\title{
Convective Cloud Bands Downwind of Mesoscale Mountain Ridges
}

\author{
DANIEL J. KIRSHBAUM \\ Department of Atmospheric and Oceanic Sciences, McGill University, Montreal, Quebec, Canada \\ DAVID M. SCHULTZ \\ Centre for Atmospheric Science, School of Earth and Environmental Sciences, University of Manchester, \\ Manchester, United Kingdom
}

(Manuscript received 23 July 2018, in final form 27 September 2018)

\begin{abstract}
Elongated and quasi-stationary cloud bands capable of producing heavy precipitation have recently been observed in the lee of midlatitude mountain ridges. Herein, idealized explicit-convection simulations are used to investigate such bands. A methodical sampling of environmental parameter space reveals that the bands are favored by a multilayer upstream static-stability profile, with a conditionally unstable midlevel layer overlying an absolutely stable surface-based layer. Such profiles promote the formation of leeside hydraulic jumps, with deep upright ascent that initiates elevated moist convection. Over smooth ridges, isolated bands develop past each ridge end due to a local superposition of cross-barrier and along-barrier pressure gradients. This superposition enhances leeside vertical displacements compared to parcels traversing the ridge midsection. In the Northern Hemisphere, the Coriolis force favors the left band over the right band (relative to the incoming flow) due to opposite-signed relative-vorticity perturbations past the two ridge ends. Whereas the negative vorticity anomaly past the left end enhances forcing for ascent, the positive vorticity anomaly past the right end suppresses it. For the environmental flows considered herein, the simulated bands are the most persistent over medium-height (1.5-km high) ridges, which force stronger leeside ascent than taller or shorter ridges. Over more rugged terrain, additional bands form past deep gaps or valleys, again due to a local superposition of horizontal pressure gradients. In contrast to some recent studies of orographic cloud bands, these simulated bands owe their existence to the release of moist static instability, indicating that neither slantwise nor inertial instability is required for their formation.
\end{abstract}

\section{Introduction}

Moist flow over orography is known to generate quasistationary convective cloud bands that locally focus precipitation and increase flash-flooding risk. Such bands have been observed past small-scale topographic features just upwind of major mountain ridges (herein termed "upwind" bands; e.g., Miniscloux et al. 2001; Kirshbaum and Durran 2005b; Kirshbaum et al. 2007a). These bands owe their existence to the release of moist static instability, initiated by surface-based flow convergence and/or elevated mountain-wave updrafts immediately past small-scale topographic features (Cosma et al. 2002; Kirshbaum et al. 2007b).

Corresponding author: Daniel J. Kirshbaum, daniel.kirshbaum@ mcgill.ca
Elongated cloud bands may also develop in the mountain lee (herein termed "downwind" bands). The majority of studies on this phenomenon have focused on isolated bands past convergent mountain wakes, where orographically blocked airstreams that divert around the ridge ends collide (e.g., Mass 1981; Andretta and Hazen 1998; Barrett et al. 2015; Scheffknecht et al. 2016). However, downwind bands may also develop within, or along the lateral edges of, the mountain wake, possibly due to different dynamical mechanisms. For example, in a recent event over the Alps, several flow-parallel cloud bands developed immediately past the ridge in deep-layer southerly flow prior to the arrival of a cold front (Siedersleben and Gohm 2016). They concluded that the bands were driven by the release of elevated inertial instability within orographically generated anticyclonic potential-vorticity (PV) banners. 


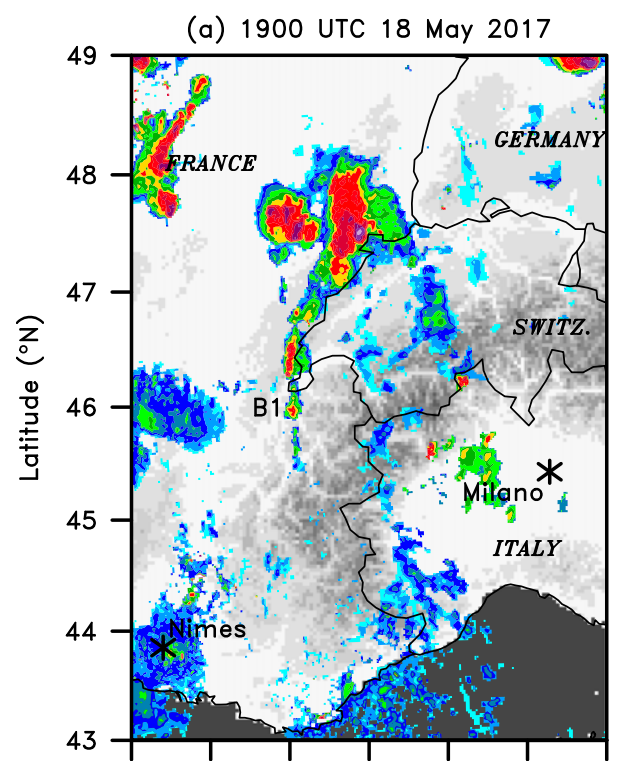

(c) 2300 UTC 18 May 2017

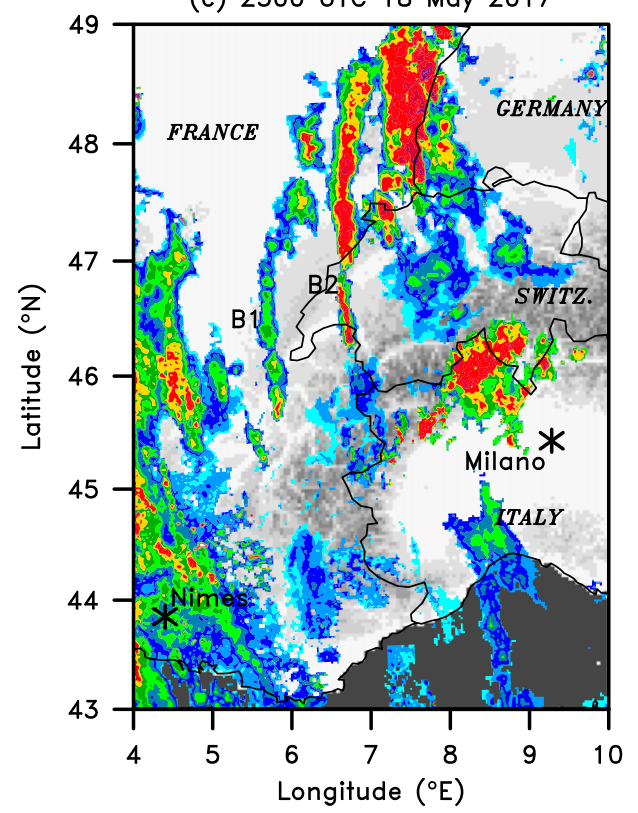

(b) 2100 UTC 18 May 2017

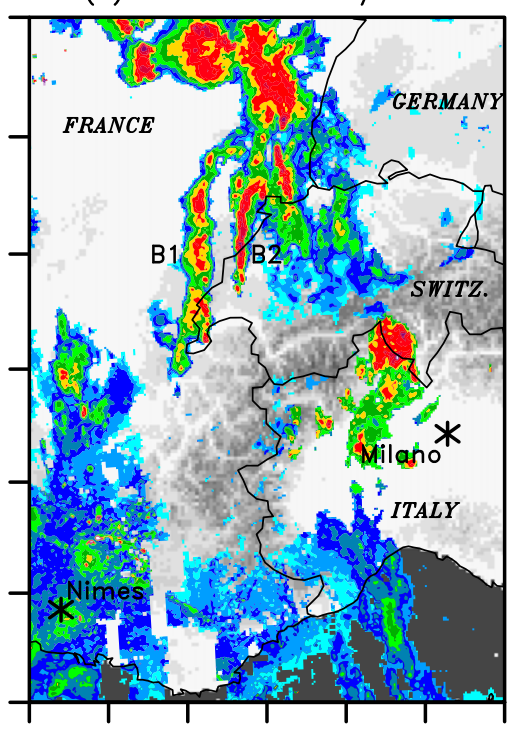

(d) 0000 UTC 19 May 2017

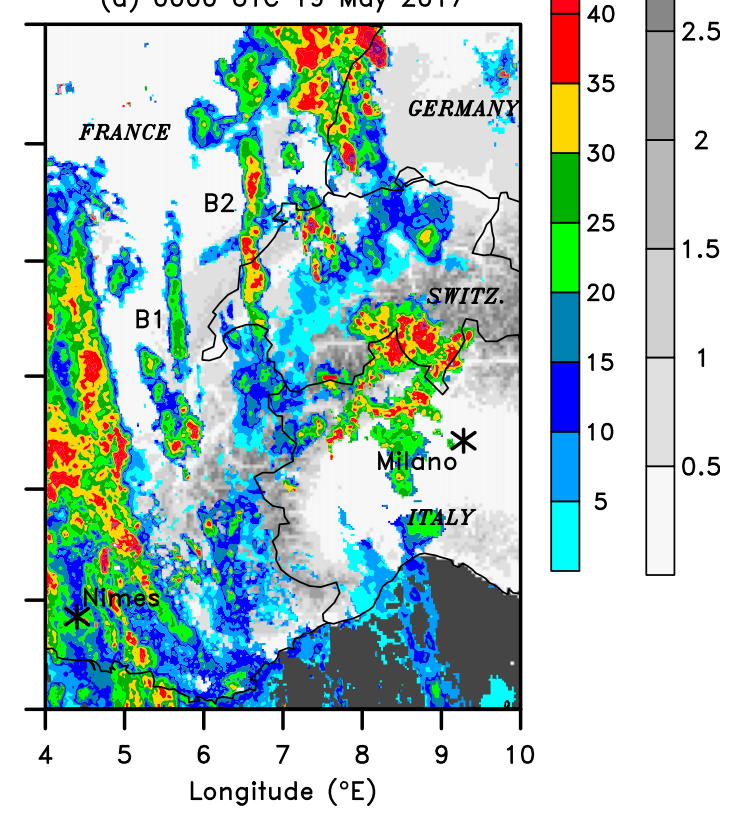

FIG. 1. Snapshots of maximum reflectivity in the vertical column from the EUMETSAT Operational Programme for the Exchange of Weather Radar Information (OPERA) European radar composite (Huuskonen et al. 2014) for selected times during the 18-19 May 2017 downwind band event.

Although the downwind bands studied by Siedersleben and Gohm (2016) produced minimal precipitation, other instances of such bands may produce heavy precipitation. One such event occurred on 18-19 May 2017, again in southerly flow over the Alps as a frontal system approached the region. The radar images in Fig. 1 indicate two prominent bands (labeled B1 and B2) that formed past deep valleys to the north of the Alps. The western band (B1) developed first but gradually weakened as the eastern band (B2) developed. B2 maintained its location and intensity for nearly $6 \mathrm{~h}$, with embedded radar-reflectivity maxima exceeding $40 \mathrm{~dB} Z$ throughout this period.

In another event in the western United States, numerous flow-parallel snowbands developed over and downwind of the Rocky Mountains (Schumacher et al. 2010, 2015). Whereas the primary band with the heaviest cumulative precipitation (around $7 \mathrm{~cm}$ of snow) was driven by frontogenesis, others (so-called minor bands) were anchored to mesoscale topographic features. These minor bands coincided with multiple mesoscale 
instabilities (inertial, symmetric, and moist-static), and the precise mechanisms responsible for their formation were not determined.

A model sounding near a band in Schumacher et al. (2010) indicated nearly dry-neutral conditions over the lowest $300 \mathrm{hPa}$ [and modest convective available potential energy (CAPE)]. Soundings taken upstream of the Rockies exhibited similar characteristics (not shown), with nondimensional mountain heights $\left(M=N h_{m} / U\right.$, where $N$ is the bulk subcrest dry Brunt-Väisälä frequency, $U$ is the mean subcrest cross-barrier wind speed, and $h_{m}=2.5 \mathrm{~km}$ is an approximate mean ridge height) well below unity. Given that $M$ broadly distinguishes between blocked $(M>1)$ and unblocked $(M<1)$ flow (e.g., Smith 1989), the incoming flow likely surmounted the Rockies with ease.

In contrast, the upstream soundings in Siedersleben and Gohm (2016) exhibited a multilayered stability profile with a nearly moist-neutral layer at midlevels $(\sim 3-6 \mathrm{~km})$ in between two absolutely stable layers. For this case $M \approx 5$ (again assuming $h_{m}=2.5 \mathrm{~km}$ ), suggesting upstream blocking and flow splitting. For the 18-19 May 2017 event, soundings were obtained at 0000 UTC 19 May at Nimes, France, and Milano, Italy (see Fig. 1 for city locations). The bands formed approximately halfway between these two sites (in the zonal direction), so we present both soundings in Fig. 2. A multilayer stability profile similar to that from Siedersleben and Gohm (2016), with weak midlevel stability between two more stably stratified layers, was observed at Nimes (Fig. 2a). The Milano sounding is similar except that the $800-900-\mathrm{hPa}$ layer is less stable, the tropopause is higher, and the mid- to upperlevel winds are weaker (Fig. 2b). Both soundings have small CAPE (142 and $56 \mathrm{~J} \mathrm{~kg}^{-1}$, respectively) and convective inhibition (CIN; 12 and $0.3 \mathrm{~J} \mathrm{~kg}^{-1}$ ), with positive parcel buoyancy in the 800-600-hPa layer. Again, $M \gg 1$ in both soundings, suggesting low-level flow blocking by the Alps. This expectation is consistent with $1000-800-\mathrm{hPa}$ wind veering in the Milano sounding, which reflects westward deflection of the incoming flow.

Based on the handful of events mentioned above, the environmental conditions, morphology, and dynamics of downwind bands may vary from case to case. Thus, multiple physical mechanisms may be responsible for producing them. In this study, we aim to build insight into some of these mechanisms via idealized numerical simulation. In particular, we focus on bands that form within or along the lateral edges of the mountain wake (e.g., Fig. 1), which have received little previous attention. Through careful control of the initial state and orographic forcing, we simulate downwind bands broadly resembling those previously observed, and physically interpret the mechanisms behind them. (a) Nimes
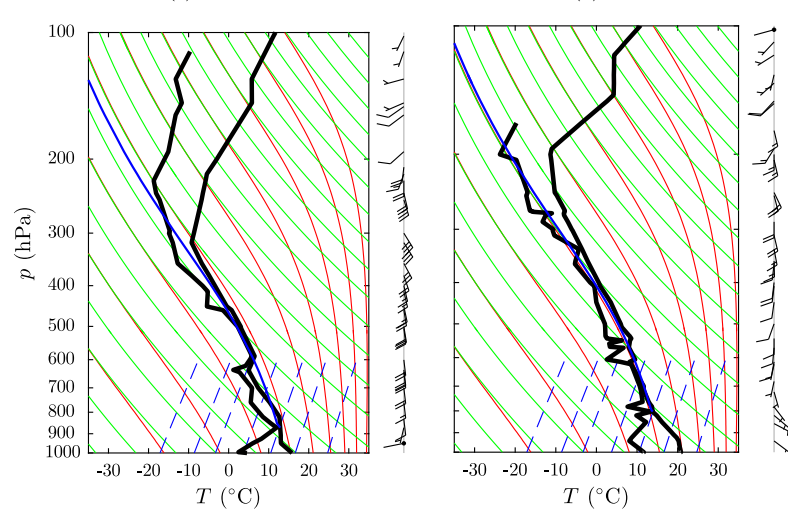

FIG. 2. Skew $T-\log p$ profiles at 0000 UTC 19 May 2017 at (a) Nimes and (b) Milano. Full wind barbs represent $10 \mathrm{~m} \mathrm{~s}^{-1}$, and blue solid lines represent the temperature of the most unstable (the one with largest $\theta_{e}$ over the lowest $400 \mathrm{hPa}$ ) adiabatically lifted parcel.

The remainder of this article is organized as follows. Section 2 describes the model configuration and initial conditions. Section 3 determines environmental conditions that favor simulated downwind bands through a methodical exploration of environmental parameter space. Section 4 uses the mass continuity equation and horizontal divergence tendency equation to explain the physical processes responsible for the bands. Section 5 considers additional sensitivities of band formation to ridge height, small-scale terrain features, and horizontal grid spacing. Section 6 discusses the similarity of the simulated bands to observed bands, and section 7 presents a summary and concluding remarks.

\section{Model setup}

The simulations use the Advanced Research version of the Weather Research and Forecasting Model, version 3.7 (WRF; Skamarock et al. 2008), in an idealized, nested-grid configuration, with a horizontal grid spacing of $\Delta_{h}=5 \mathrm{~km}$ on the outer grid and $\Delta_{h}=1.67 \mathrm{~km}$ on the inner nest (Fig. 3). The latter is sufficient to explicitly represent, if not fully resolve, downwind bands with characteristic widths of $\sim 10 \mathrm{~km}$. This $\Delta_{h}$ provides the highest affordable resolution for the many experiments considered herein, given available computational resources. The sensitivity of the simulated flows to the choice of $\Delta_{h}$ is briefly examined in section 5 .

Although open/radiative lateral boundary conditions (LBCs) are often used in idealized simulations of orographic flows, such LBCs caused undesirable domain-wide pressure losses that complicated physical interpretation. As an alternative, periodic LBCs are used in $x$ along with rigid LBCs in $y$, thus representing a 


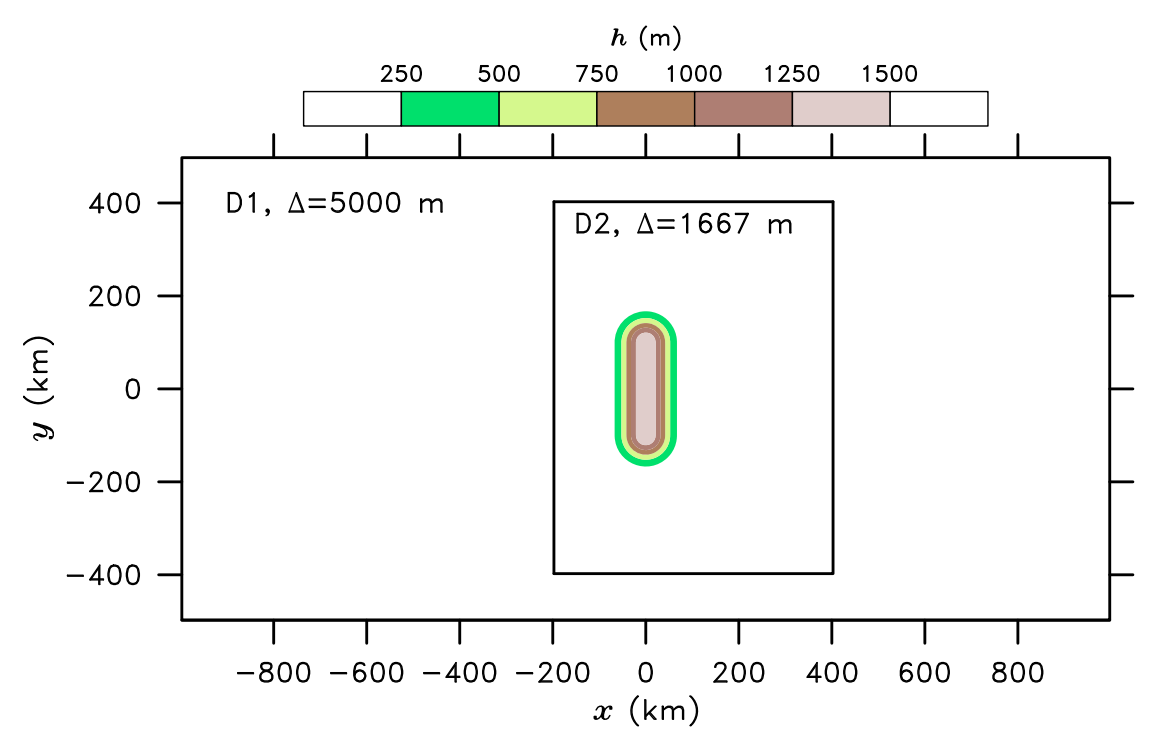

FIG. 3. Model grid configuration and terrain height.

periodic zonal channel flow. The horizontal domain is sufficiently large $\left(L_{x}=2000 \mathrm{~km}\right.$ by $\left.L_{y}=1000 \mathrm{~km}\right)$ that mountain-induced flow perturbations do not recirculate through it over the 12-h model integration. This configuration is particularly useful for the simulations of baroclinic flows in section 3 , where the flow is aperiodic in $y$. In the vertical, 101 stretched hydrostatic-mass levels are used with a model top at $L_{z}=20 \mathrm{~km}$, giving a nominal grid spacing of $80 \mathrm{~m}$ at the surface and 56 levels below $6 \mathrm{~km}$. A Rayleigh damping layer is used to absorb gravity waves over the uppermost $6 \mathrm{~km}$.

The default mesoscale mountain ridge is given by

$h_{s}(x, y)=\left\{\begin{array}{cl}h_{m} \exp \left(-\frac{x^{2}}{a^{2}}\right), & |y| \leq b \\ h_{m} \exp \left\{-\frac{x^{2}}{a^{2}}-\frac{[y-\operatorname{sgn}(y) b]^{2}}{a^{2}}\right\}, & |y|>b\end{array}\right.$,

where $h_{m}=1.5 \mathrm{~km}, a=50 \mathrm{~km}$, and $b=100 \mathrm{~km}$. This function broadly represents medium-height midlatitude mountain ridges like the Oregon Cascades, the Italian Appenines, and the Australian Alps. The sensitivity of the model results to $h_{m}$ is evaluated in section 5 .

Physical parameterizations include the WRF singlemoment 6-class cloud microphysics scheme (WSM6; Hong et al. 2006) and 1.5-order turbulent kinetic energy (TKE) subgrid turbulence closure (Janjić 1994). For simplicity, an unheated, free-slip surface is used with no boundary layer scheme. The Coriolis force is either neglected (nonrotating) or implemented on a midlatitude $f$ plane with $f_{0}=2 \Omega \sin \left(\phi_{0}\right)$, where $\phi_{0}=45^{\circ} \mathrm{N}$ (rotating).
Horizontal and vertical advection are fifth-order and third-order, respectively, with positive-definiteness applied to moisture, subgrid TKE, and other scalar variables.

The initial state is determined by a few parameters: a thermodynamic profile at the meridional domain center point $(y=0)$ consisting of a temperature $T_{0}$ at $z=0$, a uniform relative humidity $(\mathrm{RH})$ of $80 \%$, a vertical profile of the Brunt-Väisälä frequency $N(z)$, and a horizontally uniform westerly wind profile $U(z)=U_{0}+U_{z} z$, where $U_{0}=10 \mathrm{~m} \mathrm{~s}^{-1}$ is the surface wind and $U_{z}$ is the vertical shear. In the nonrotating simulations, the initial flow is horizontally homogeneous, with the central profile applied over the entire domain. In the rotating simulations, the meridional variations of temperature, pressure, and water vapor mixing ratio are constrained by thermal wind balance in a moist atmosphere [as in Kirshbaum et al. (2018)], with RH held fixed at $80 \%$. In the absence of terrain, this balanced flow remains steady indefinitely.

To seed mesoscale instabilities, a 3D field of uniformly distributed random temperature perturbations with a maximum amplitude of $0.1 \mathrm{~K}$ is added to the initial state. Although the simulated flows never reach a full steady state due to the prolonged development of the mountain wake, transient development weakens after $\sim 6 \mathrm{~h}$ of time integration, so for computational efficiency we limit the model integration to $12 \mathrm{~h}$.

\section{Determining suitable environmental conditions for band formation}

We begin with a methodical sampling of a few of the environmental parameters defined above to assess if certain combinations of these parameters are particularly 
favorable for the development of downwind bands. Rather than making any specific assumptions about the environmental conditions supporting the bands, we begin our inquiry using a very simple upstream flow and gradually add (or reduce) complexity as needed. The choice of which complexities to add is informed by the environmental conditions during observed downwind-band events. For reference, parameter choices for all of the simulations conducted herein are listed in Table 1.

\section{a. Single layer, absolutely stable}

The first simulation, called N012, is initialized with $T_{0}=283 \mathrm{~K}, U_{z}=1.5 \times 10^{-3} \mathrm{~s}^{-1}, N=0.012 \mathrm{~s}^{-1}$ uniformly, and no Coriolis force. These settings yield an absolutely stable initial thermodynamic profile that inhibits the development of moist convection (Fig. 4a). The cloud water path,

$$
\mathrm{CWP}=\int_{h_{s}}^{L_{z}} \rho_{d} q_{h} d z,
$$

where $\rho_{d}$ is the dry-air density and $q_{h}$ is the full hydrometeor mixing ratio, is overlaid on the lowest-model-level pressure perturbation (relative to the hydrostatically balanced initial state) and wind vectors of N012 in Figs. 5a-c. By $6 \mathrm{~h}$ of time integration, clouds develop over both the windward slope and in the lee (Fig. 5a). Typical of orographic flows, the windward and lee slopes are respectively characterized by perturbation high and low pressure. Over the remainder of the simulation, an upstream-propagating bore develops on the windward side with an arc-shaped cloud at its leading edge, similar to that found in previous simulations of orographically blocked flows (e.g., Galewsky 2008). Such blocking is expected given a nondimensional mountain height of $M=1.6$ in this case (Table 1). Two lee vortices, antisymmetric about $y=0$, also propagate downstream in the wake (Figs. 5b,c). The cloud field gradually expands meridionally over time, as the mountain wave over the ridge propagates laterally with height. No elongated flow-parallel clouds are evident.

A second simulation, N012-COR, is identical to N012 except for including rotation and the meridional density and pressure gradients required for thermal-wind balance. The resulting flow and cloud patterns resemble the corresponding nonrotating simulation at $6 \mathrm{~h}$ but develop some differences later (Figs. 5d-f). Unlike the symmetric windward flow splitting about $y=0$ in N012, the blocked incoming flow detours predominantly to the north, with a separation point near the southern ridge end. As in the 2D simulations of Pierrehumbert and Wyman (1985), this geostrophic adjustment limits the development of the upstream-propagating bore. It also promotes stronger terrain-forced ascent, as well as a correspondingly stronger leeside wave response, over the northern half of the ridge, which shifts the cloud field northward [again similar to Galewsky (2008)]. The wake flow becomes dominated by the northern anticyclonic vortex (Fig. 5f), with strong flow reversal to its south, and the northern part of the cloud mass elongates toward the east-northeast due to midlevel mountain-wave ascent. Although this feature has a banded appearance, it still does not resemble observed downwind bands (e.g., Fig. 1).

Recall that the release of inertial instability was cited as a potential mechanism for downwind bands (Schumacher et al. 2010; Siedersleben and Gohm 2016). Although such instability was precluded in the nonrotating N012 case, it is possible in the rotating N012-COR case. We assess its presence (or lack thereof) in the latter by adding the zero contour of low-level (0-3-km-averaged) absolute vorticity $\zeta_{a}$ to Figs. 5d-f. Areas enclosed by the green contours possess $\zeta_{a}<0$ and thus satisfy a necessary condition for inertial instability (e.g., Holton 1972; Schultz and Schumacher 1999; Thompson et al. 2018). Our evaluation of $\zeta_{a}$ focuses on lower levels where the $\zeta_{a}$ perturbations are maximized, but the result does not strongly depend on the precise layer chosen for the evaluation (not shown). Broad areas of inertial instability are apparent over the crest, lee, and wake of the ridge, some coinciding with clouds. However, little correspondence between this instability and clouds is found over the wake region where downwind bands tend to develop.

The asymmetry of the lee vortices in N012-COR can be explained by the ageostrophic flow response to the orographic forcing. As seen in Fig. 5, the wake is characterized by a local pressure minimum, which tends to weaken the meridional pressure gradient past the northern ridge end and strengthen this gradient past the southern ridge end. As the westerly winds accelerate around the northern ridge end, they become strongly supergeostrophic and deflect southward into the wake. Meanwhile, the decelerated and subgeostrophic flow near the foot of the lee slope accelerates northward due to the meridional pressure gradient force. These inertial motions reinforce the anticyclonic vortex in the northern wake. By contrast, the accelerated westerly winds around the southern ridge end are nearly balanced by an enhanced meridional pressure gradient there, which limits their northward penetration into the wake and thereby suppresses the southern vortex.

\section{b. Single layer, conditionally unstable}

Given that elongated leeside bands failed to develop in the above absolutely stable flows, we ask whether 


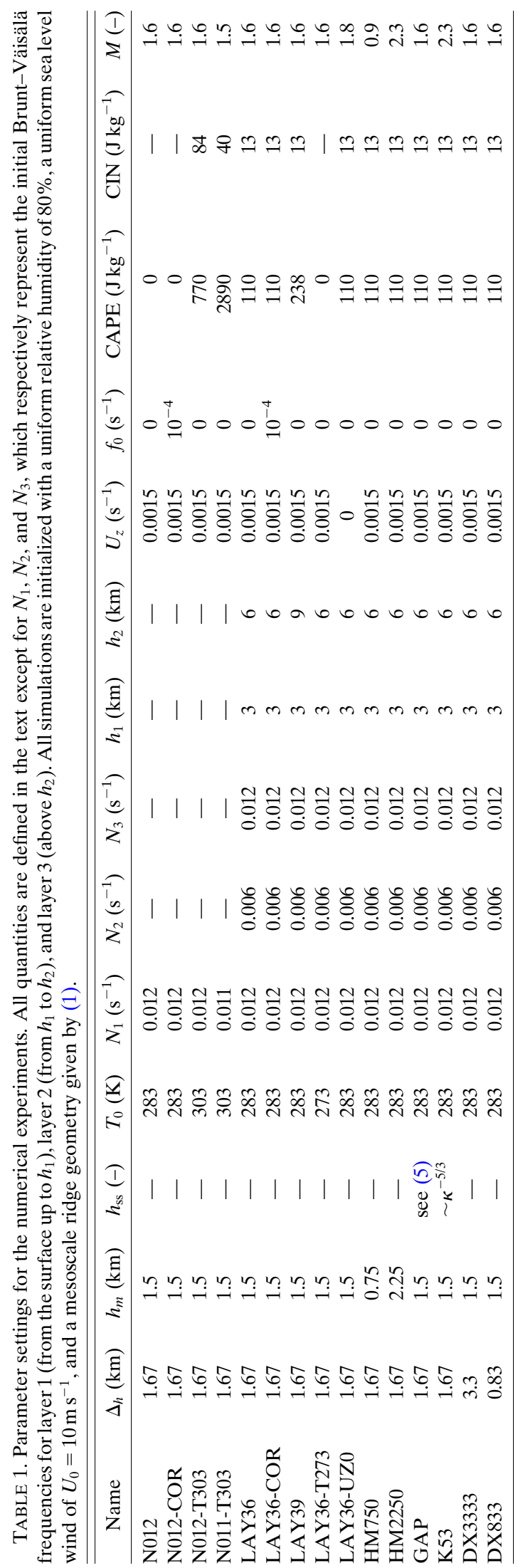


(a) N012

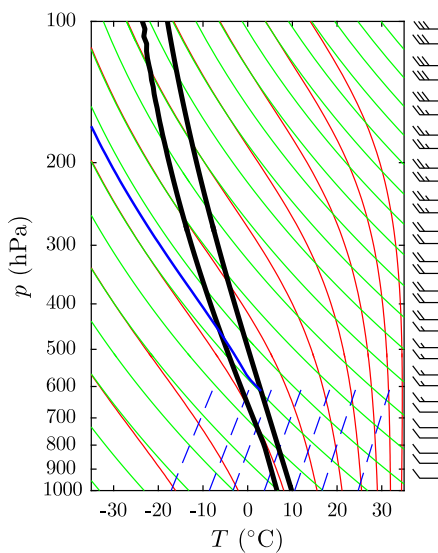

(b) N012-T303

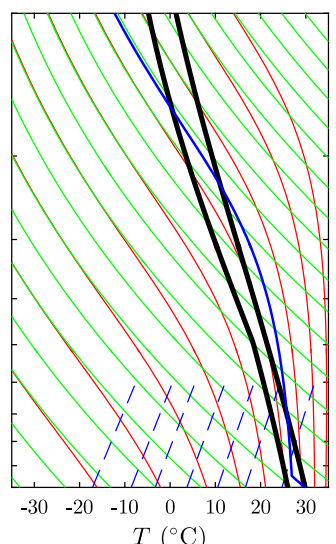

(c) LAY36

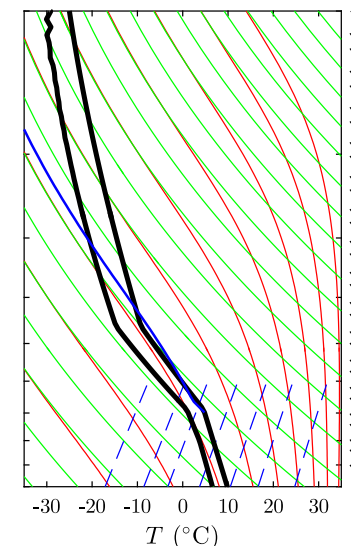

(d) LAY39

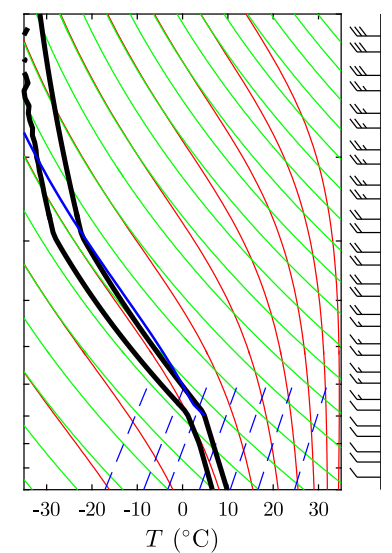

FIG. 4. Initial skew $T-\log p$ profiles for four selected simulations. Full wind barbs represent $10 \mathrm{~m} \mathrm{~s}^{-1}$, and blue solid lines represent the temperature of the most unstable (the one with largest $\theta_{e}$ over the lowest $400 \mathrm{hPa}$ ) adiabatically lifted parcel.

conditionally unstable environments are more conducive to band formation. To address this question, we consider an upstream flow identical to that in N012 except for a much higher surface temperature of $T_{0}=303 \mathrm{~K}$ (N012-T303; Fig. 4b). The consequent decrease in moist adiabatic lapse rates yields conditional instability over $1000-400 \mathrm{hPa}$, with a moderate CAPE of $770 \mathrm{~J} \mathrm{~kg}^{-1}$. However, the release of this instability is inhibited by a large CIN of $84 \mathrm{~J} \mathrm{~kg}^{-1}$, leaving a mesoscale flow pattern broadly resembling the N012 case
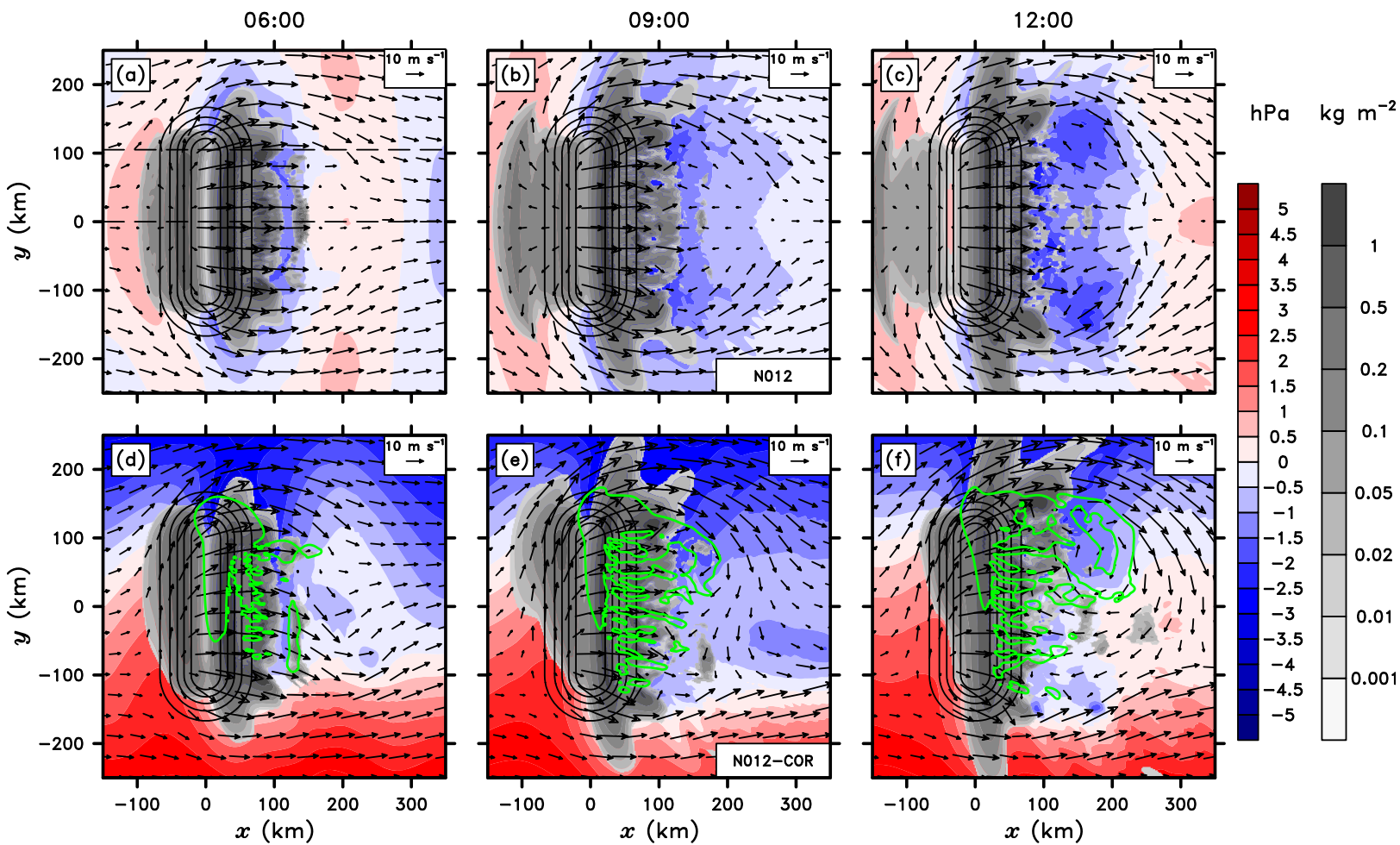

FIG. 5. Lowest-model-level pressure perturbation (color fill), wind vectors, and CWP (gray fill) for the (a)-(c) N012 and (d)-(f) N012-COR simulations. The single green contour of the rotating N012-COR simulation in (d)-(f) denotes $0-3$ - $\mathrm{km}^{-a v e r a g e d} \zeta_{a}=0 \mathrm{~s}^{-1}$; hence, areas enclosed by these contours are inertially unstable. This $\zeta_{a}$ contour is not shown in the nonrotating N012 simulations in (a)-(c) because inertial instability is precluded. Terrain is shown in solid black contours every $250 \mathrm{~m}$, and the dashed black lines in (a) indicate the locations of the vertical cross-sections shown in Figs. 9a and c. 

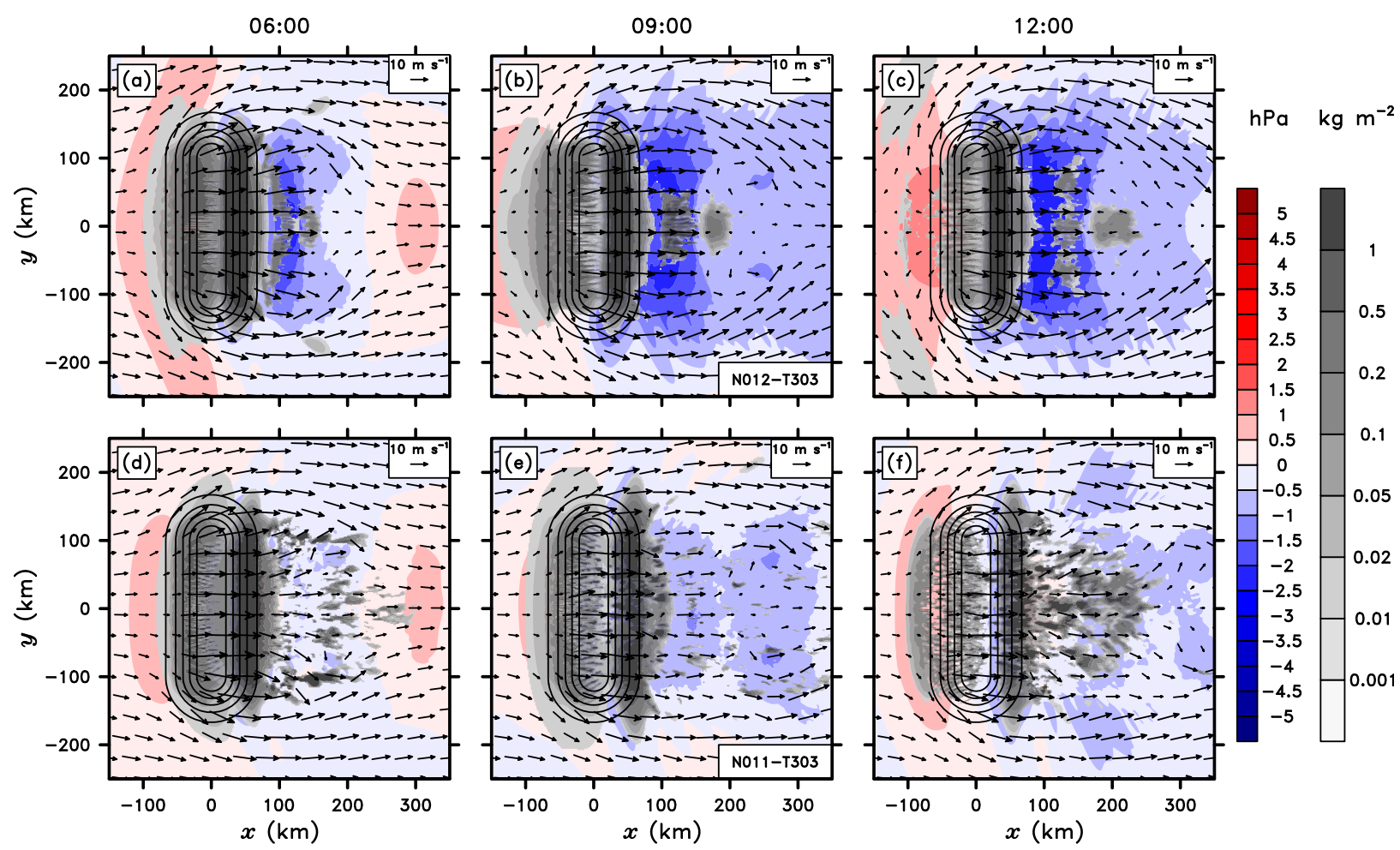

FIG. 6. As in Fig. 5, but for the (a)-(c) N012-T303 and (d)-(f) N011-T303 simulations.

(cf. Figs. 5a-c and 6a-c). One notable difference is a modest reduction in upstream blocking in N012-T303 due to a destabilized windward cap cloud, which fosters increased penetration of westerly flow into the wake.

To consider a more convective flow, we reduce $N$ to $0.011 \mathrm{~s}^{-1}$ (N011-T303), which greatly increases CAPE $\left(2890 \mathrm{~J} \mathrm{~kg}^{-1}\right)$ and reduces CIN $\left(40 \mathrm{~J} \mathrm{~kg}^{-1}\right)$. The resulting decrease in $M$ (from 1.6 to 1.5; Table 1) further weakens upstream blocking and its resulting upstream-propagating bore (Figs. 6d-f). However, because the near-surface flow still deflects around the ridge, the most unstable impinging air parcels are not directly lifted to saturation over the windward slope. This effect, coupled with elevated mountain-wave descent directly over the crest (to be shown later), limits the windward cloud tops to $\sim 6 \mathrm{~km}$. In contrast, the leeside convection is more vigorous and deeper, locally reaching above $10 \mathrm{~km}$ (not shown). Interestingly, two elongated but disorganized bands develop along the lateral edges of the wake by $6 \mathrm{~h}$ (Fig. 6d). Although these bands broadly match the description of downwind bands given in section 1 , they give way to more chaotic wake convection later (Figs. 6e,f).

\section{c. Three layers with midlevel conditional instability}

The development of two ephemeral downwind bands past the mountain edges in N011-T303 suggests that, as with upwind bands, moist instability may be a necessary ingredient for these bands to form. However, given the subsequent dissipation of the bands, the initial conditions of that simulation do not favor their persistence. Because the upstream flows in Siedersleben and Gohm (2016) and in the 18-19 May 2017 case both exhibited a multilayer tropospheric stability profile with reduced midlevel stability, we turn to a three-layer flow with a stable lower layer $\left(N=0.012 \mathrm{~s}^{-1}\right)$ from 0 to $3 \mathrm{~km}$, a weakly stable middle layer $\left(N=0.006 \mathrm{~s}^{-1}\right)$ from 3 to $6 \mathrm{~km}$, and a stable upper layer $\left(N=0.012 \mathrm{~s}^{-1}\right)$ (Fig. 4c). All other parameters in this simulation (LAY36) are identical to those in N012, including $M$ (Table 1). The maximum CAPE is a modest $110 \mathrm{~J} \mathrm{~kg}^{-1}$, with a corresponding CIN of $13 \mathrm{~J} \mathrm{~kg}^{-1}$, for the most unstable parcel based at $3 \mathrm{~km}$.

The LAY36 cloud field contains two downwind bands past the ridge edges that develop by $6 \mathrm{~h}$ and persist until the end of the simulation (Figs. 7a-c). Because these bands form past the ridge edges, they are henceforth termed "edge bands." Before interpreting their physical mechanisms in detail (section 4), we briefly explore some environmental sensitivities of these bands. First, as in N012-COR, we perform a simulation with the Coriolis force included and a thermal-wind-balanced initial state (LAY36-COR). Although the early development of the cloud field in this simulation resembles that in its nonrotating counterpart (Fig. $7 d$ ), the northern edge band 

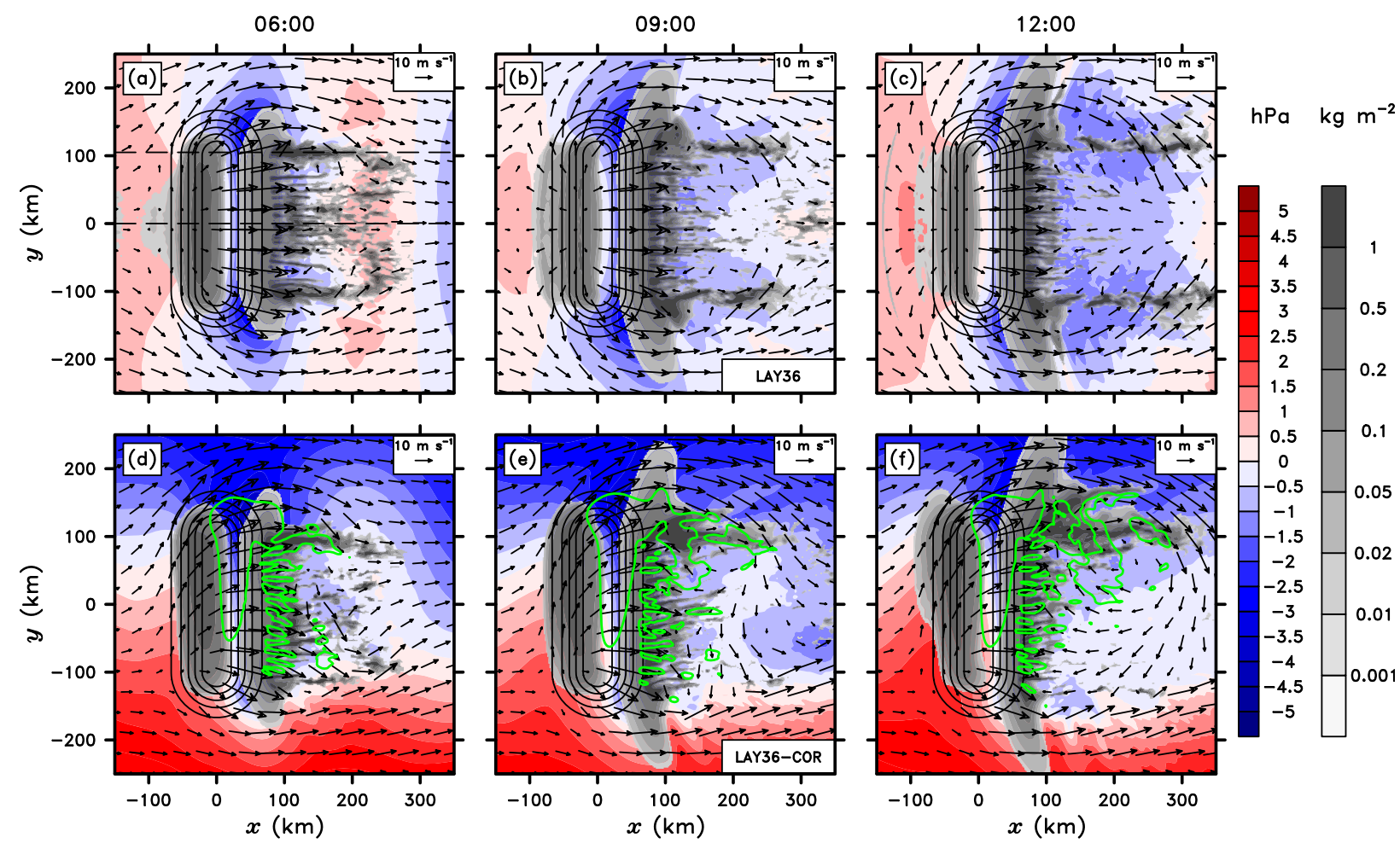

FIG. 7. As in Fig. 5, but for the (a)-(c) LAY36 and (d)-(f) LAY36-COR simulations. The dashed black lines in (a) indicate the locations of the vertical cross-sections shown in Figs. 9b and d.

ultimately dominates over the southern one (Figs. 7e,f). This finding suggests that, at least in the midlatitudes, the left edge band (from the perspective of the impinging flow) is preferred over the right one. An explanation for this preference is provided in section 4 .

Similar to N012-COR, the anticyclonic lee vortex in the northern wake of LAY36-COR progressively dominates over the cyclonic vortex in the southern wake (Fig. 7f). Also, most of the area occupied by the northern band coincides with low-level $(0-3 \mathrm{~km})$ inertial instability. Although this finding is consistent with the hypothesis of Siedersleben and Gohm (2016), the fact that similar bands still formed in the absence of inertial instability (in LAY36) suggests that this instability is either incidental or serves to reinforce, rather than initiate, the edge bands. Moreover, some of this inertial instability may have been generated by the convection itself (e.g., Schultz and Knox 2007).

To further evaluate the environmental sensitivities of the bands, we conduct a few additional tests, all using nonrotating atmospheres for simplicity. We begin with a simulation that is identical to LAY36 except that the depth of the middle layer with $N=0.006 \mathrm{~s}^{-1}$ is increased from 3 to $6 \mathrm{~km}$, so that it spans from 3 to $9 \mathrm{~km}$ (LAY39; Fig. 4d). Although the most unstable CIN is unchanged, the most unstable CAPE increases to $238 \mathrm{~J} \mathrm{~kg}^{-1}$. The resulting cloud field is similar to that of LAY36 except that the edge bands, along with the more disorganized convection past the central ridge, are more prominent and intense (Figs. 8a-c). A similar intensification is found if, instead of deepening the middle layer, its stability is reduced. Also, in experiments where the middle layer is extended all the way to the domain top, thus leaving only a two-layer flow, the bands persist (not shown). Hence, the stable upper layer is unnecessary for edge-band formation.

To evaluate whether moist instability is required for the edge bands to form, we conduct a simulation identical to LAY36 except that $T_{0}$ is lowered from 283 to $273 \mathrm{~K}$ (LAY36-T273). This modification has no impact on the dry stability, which is still governed by the same three-layer $N(z)$ profile. However, it fully eliminates the moist instability, such that CAPE is initially everywhere zero. The surface pressure pattern in this simulation is similar to that in LAY36 except for enhanced pressure perturbations due to reduced cloud latent heat release (Figs. 8d-f). However, no edge bands develop, confirming that moist instability is a necessary condition for the development of the bands in LAY36 (Figs. 7a-c) and LAY39 (Figs. 8a-c).

Finally, to examine the role of basic-state wind shear (and hence baroclinicity) in band formation, we conduct a 

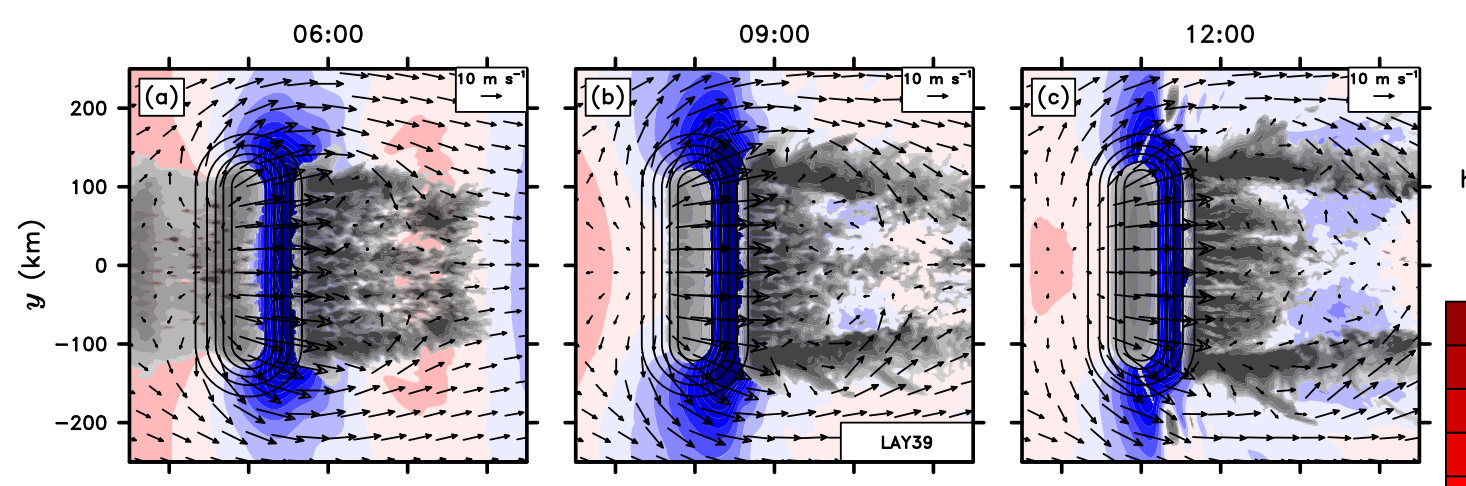

$\mathrm{hPa} \quad \mathrm{kg} \mathrm{m}^{-2}$
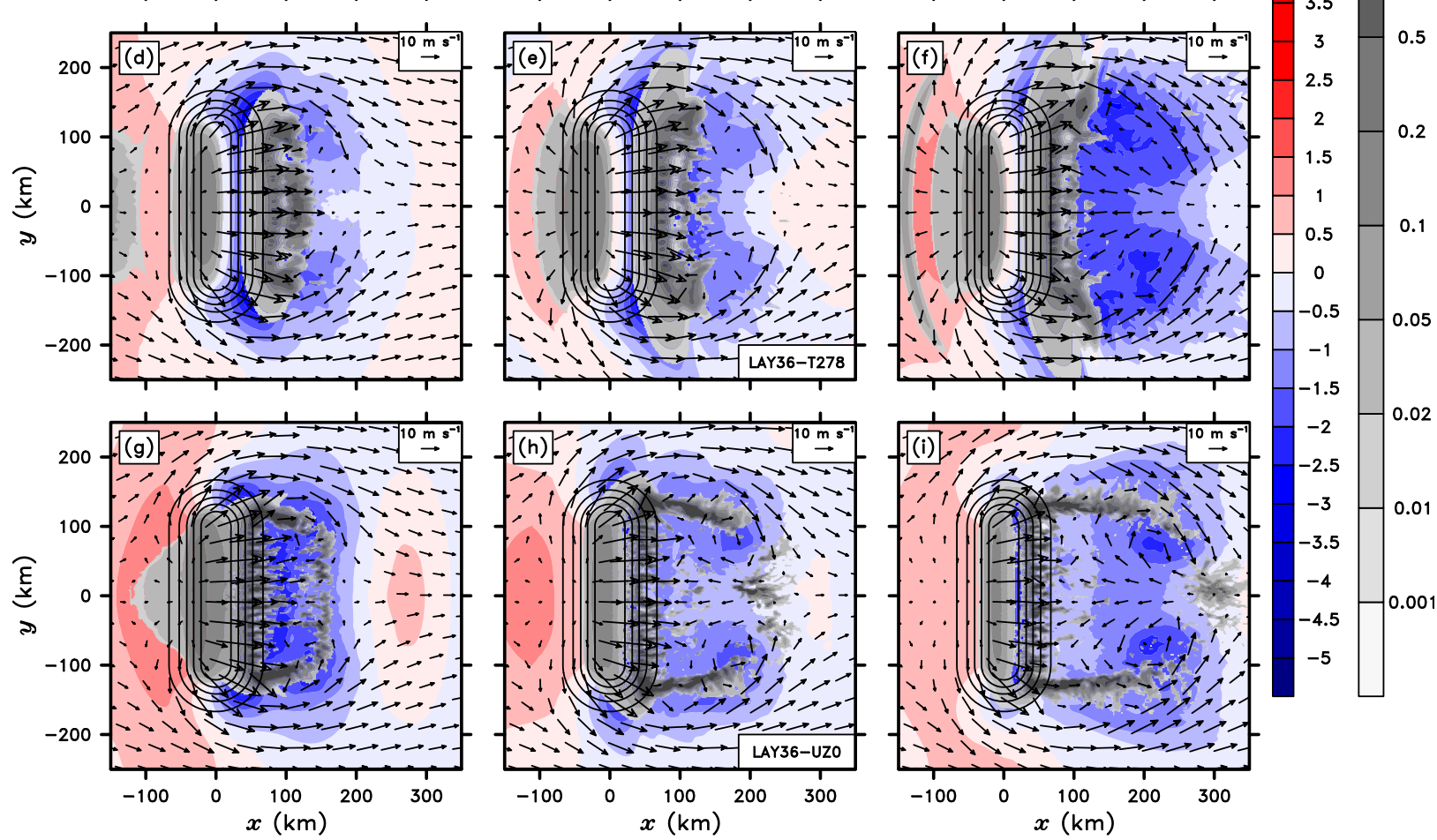

FIG. 8. As in Fig. 5, but for (a)-(c) LAY39, (d)-(f) LAY36-T278, and (g)-(i) LAY36-UZ0 simulations.

simulation identical to LAY36 except that the wind profile is unsheared $\left(U_{z}=0 \mathrm{~s}^{-1}\right)$, so that the initial winds are uniformly $U=U_{0}=10 \mathrm{~m} \mathrm{~s}^{-1}$ (LAY36-UZ0). The reduced winds aloft increase $M$ slightly to a value of 1.8 , which marginally increases the upstream blocking. Although the resulting leeside pressure pattern in this case undergoes less meridional expansion than that of the vertically sheared LAY36 simulation, the downwind bands past the ridge ends still develop, and are arguably even better defined (Figs. 8g-i). Given that vertical wind shear generally serves to organize convection in the along-shear (here west-east) direction (e.g., Asai 1970), the improved band organization in the absence of such shear may be surprising. However, convection anchored to orography may organize into bands even without basicstate vertical shear, due to their streamwise elongation past a fixed initiation point (Kirshbaum and Durran 2005a; Kirshbaum et al. 2007a).

\section{d. Vertical cross sections}

To convey the key differences in leeside vertical motions between continuously stratified and multilayer atmospheres, vertical-zonal cross sections of vertical velocity $w$, ice-liquid water potential temperature $\theta_{i l}$, and hydrometeor mixing ratio $q_{h}$ are compared at $6 \mathrm{~h}$ from N012 and LAY36. Because $\theta_{i l}$ is conserved for moist adiabatic processes, its contours roughly reflect flow streamlines. Two cross sections are considered, one along the ridge centerline $(y=0 \mathrm{~km})$ and the other near the northern end of the ridge, passing through a downwind band in LAY36 ( $y=105 \mathrm{~km}$; see Fig. 7a). Although the flow in the former cross section is quasi-2D in $x$ and $z$, 

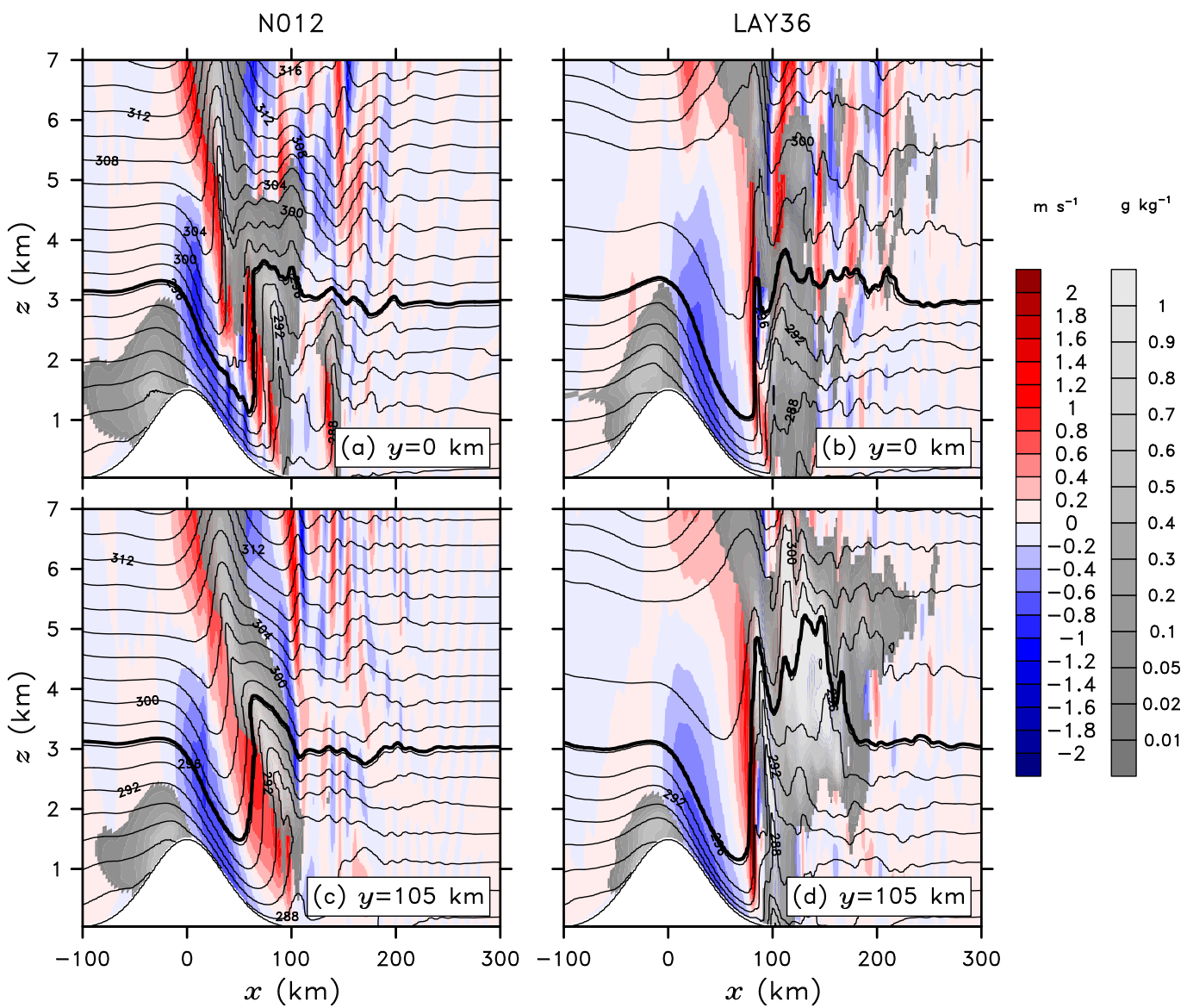

FIG. 9. Vertical-zonal cross sections of $w$ (color fill), $q_{h}$ (gray fill), and $\theta_{i l}$ (black contours) for the (left) N012 and (right) LAY36 simulations at $6 \mathrm{~h}$ and (a),(b) $y=0$ and (c),(d) $y=105 \mathrm{~km}$ (the latter through a downwind band of LAY36), respectively (see Fig. 7a). The thick black line is the $\theta_{i l}$ surface based at an initial height of $3 \mathrm{~km}$.

lateral flow deflection causes some meridional motion into and out of the plane of the latter cross section. This motion decays above crest level.

Along the ridge centerline of the N012, an upstreamtilted mountain wave develops with strong loweratmospheric $(z<5 \mathrm{~km})$ descent over the crest followed by gravity wave breaking in the lee (Fig. 9a). Based on the $\theta_{i l}$ contours, low-level air parcels that undergo rapid descent over the lee slope are returned to their original heights by the leeside updraft. A similar pattern is found at $y=105 \mathrm{~km}$ (Fig. 9c), except that mid- to upper-level streamlines (e.g., the thick black isentrope originating at $3 \mathrm{~km}$ ) are lifted up to $1 \mathrm{~km}$ beyond their original heights by the leeside updraft. In conditionally unstable flows like that in N011-T303, such lifting may suffice to initiate cumulus convection. However, such convection would struggle to survive the sharp downdraft just downwind, which forces the air parcels back down to $\sim 3 \mathrm{~km}$.

The LAY36 case also exhibits strong leeside descent followed by a sharp updraft (Fig. 9b). However, rather than tilting upstream with height, the lee updraft is nearly vertically upright. This response is qualitatively similar to that of two-layer orographic flows with stronger stability in the lower layer (Durran 1986; Durran and Klemp 1987), which behave analogously to hydraulic flow over a ridge. Over the windward slope, the lower $(0-3 \mathrm{~km})$ layer acts like a subcritical flow dominated by the pressuregradient force. As this layer accelerates over the crest under a forward pressure gradient force, the nonlinear advection strengthens until it exceeds the pressure gradient acceleration, which renders the latter incapable of decelerating the flow in the lee. This behavior is similar to a hydraulic layer transitioning from subcritical to supercritical over the crest, accelerating and thinning continuously until breaking within a leeside hydraulic jump. Above this jump, deep and nearly upright ascent occurs within the weakly stratified middle layer.

The vertically aligned leeside updraft in LAY36 simultaneously lifts the entire moist-unstable 3-6-km layer to saturation. Cross sections at $y=0 \mathrm{~km}$ and 


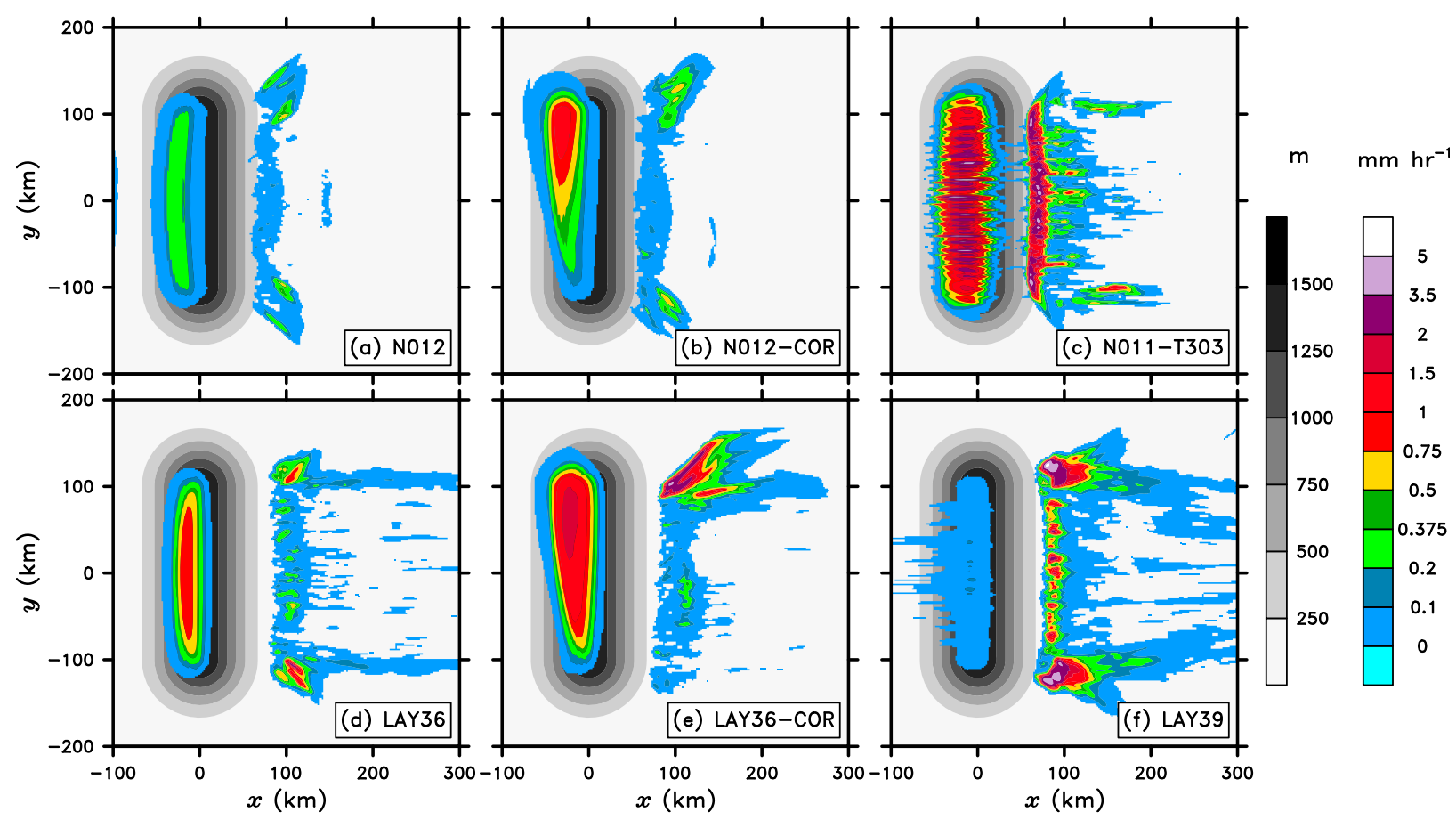

FIG. 10. Comparison of 6-12-h mean precipitation rates for selected simulations.

through the band $(y=105 \mathrm{~km})$ differ greatly, in that the latter exhibits much greater lifting within the hydraulic jump (cf. Figs. 9b and 9d). Although this enhanced displacement past the ridge end is consistent with the N012 case, its amplitude is larger and the subsequent downward displacement is insufficient to return the parcels back to their LFC. As a result, elevated moist convection is initiated past the ridge ends. The vertical structure of the band is qualitatively similar to that of the windward bands studied by Kirshbaum and Durran (2005a) and Kirshbaum et al. (2007a), except that these bands initiate along the edges of the hydraulic jump rather than past small-scale terrain features.

\section{e. Precipitation}

The differences in leeside cloud patterns illustrated above lead to differing surface precipitation distributions, which are averaged over $6-12 \mathrm{~h}$ for selected simulations in Fig. 10. The N012 simulation produces light precipitation over the windward slope and lee, with no evidence of downstream banding (Fig. 10a). A similar pattern is found in N012-COR, except that the precipitation is enhanced over the northern half of the ridge (Fig. 10b). Over the windward slope, this south-north asymmetry is caused by leftward (southerly) flow deflection, as seen in Figs. 5d-f. Because of a substantial cross-barrier component of the Coriolis force, some of this deflected flow crosses the northern half of the ridge to locally increase precipitation there (Fig. 10b). The precipitation distribution is more symmetric in the lee except for a modest enhancement in coverage past the northern ridge end.

Heavier precipitation is concentrated over the windward slope and within the leeside hydraulic jump in N011-T303, with weaker, scattered precipitation in the mountain wake (Fig. 10c). The two ephemeral cloud bands along the wake edges (Fig. 6d) also produce substantial precipitation. However, because of their short durations, the time-averaged precipitation rates of these bands are similar to that of the disorganized convection within the central wake.

A more banded leeside precipitation distribution is evident in LAY36, where localized precipitation maxima exceeding $1 \mathrm{~mm} \mathrm{~h}^{-1}$ form past each of the ridge ends (Fig. 10d). These regions coincide with the aforementioned deep layer of upright ascent at the lateral edges of the hydraulic jump (Fig. 9d). Lighter precipitation occurs along the central part of the hydraulic jump and along the wake edges, the latter aligning with the edge bands in Fig. 7. Because of their shallowness and lack of hydrometeor mass, these bands fail to produce heavy precipitation. The inclusion of rotation in LAY36-COR induces meridional asymmetry, with the heaviest precipitation over the northern windward slope and in the northern edge band (Fig. 10e).

Finally, the precipitation in LAY39 is similar to that in LAY36 except that the windward precipitation is 
reduced and the edge bands produce more precipitation (Fig. 10f). The former stems from a more hydraulic response characteristic of a two-layer atmosphere (e.g., Smith et al. 1997), wherein flow streamlines above crest level tend to descend, rather than ascend, over the crest. The increased edge-band precipitation is a direct consequence of the deeper cloud-bearing layer.

\section{Physical interpretation}

A necessary condition for convective initiation within the conditionally unstable $3-6-\mathrm{km}$ layer is that air parcels breach their level of free convection (LFC). In LAY36, the most unstable parcels originate at the base of this layer, with an LFC of around $3750 \mathrm{~m}$. To estimate the vertical displacements of these air parcels, we determine the $\theta_{i l}$ value corresponding to an initial height of $3 \mathrm{~km}$ at each meridional grid point $\left(\theta_{i l}\right)_{r}$, and then evaluate the height of this "reference", surface $\left(h_{r}\right)$ over time. In the initially horizontally homogeneous nonrotating simulations, $\left(\theta_{i l}\right)_{r}$ is a constant, whereas in the meridionally varying rotating simulations, $\left(\theta_{i l}\right)_{r}$ is a function of $y$. The layer between the ground $\left(z=h_{s}\right)$ and $h_{r}$ is henceforth termed the "sub band" layer.

The Boussinesq mass-continuity equation, integrated from the surface $\left(z=h_{s}\right)$ to $z=h_{r}$, may be written

$$
w_{r}=w_{s}-\int_{h_{s}}^{h_{r}} \nabla \cdot \mathbf{u}_{h} d z
$$

where $\mathbf{u}_{h}$ is the horizontal wind vector, $w_{r}=w\left(x, y, h_{r}\right)$, and $w_{s}=w\left(x, y, h_{s}\right)$. Comparison of the Boussinesq and anelastic mass continuity equations revealed small differences between the two, so we use the former. Based on (3), vertical motion at $h_{r}$ (or $\left.w_{r}\right)$ is governed by $w_{s}$, which is nearly zero in the mountain wake, and the vertically integrated horizontal convergence between $h_{s}$ and $h_{r}$. Because the two edge bands are nearly symmetric and become established by around $6 \mathrm{~h}$ (Fig. 7a), we analyze various time-averaged quantities relevant to (3) for the northern band over 6-9h.

Both the sub-band horizontal convergence and $w_{r}$ are maximized along the hydraulic jump at $x \approx 80 \mathrm{~km}$ (Figs. 11a,b), which aligns with the western edge of the wake. The reference-surface height $h_{r}$ is locally maximized within the boxed region near the northern end of this line (Fig. 11c) due to two factors. First, differences in terrain-forced descent over the lee slope cause $h_{r}$ to vary meridionally along the upstream edge of the boxed region (Fig. 11c). Whereas the low-level flow over the ridge midsection is mostly divergent and strongly descending (leading to small $h_{r}$ ), the low-level flow over the ridge ends is convergent and only weakly descending (leading to larger $h_{r}$ ) (Figs. 11a,c). Second, along the hydraulic jump, both convergence and $w_{r}$ are maximized within the boxed region (Figs. 11a,b). Thus, airstreams crossing the ridge ends experience less leeslope descent, and stronger ascent within the hydraulic jump, than those crossing the ridge midsection (Fig. 11c). This enhanced trajectory-integrated ascent locally eliminates the CIN and initiates the cumulus cloud band (Fig. 11d).

To explain the enhanced ascent past the ridge ends, we consider the Boussinesq horizontal divergence $(\delta)$ budget

$$
\delta_{t}=\underbrace{-(\mathbf{u} \cdot \nabla) \delta}_{\mathrm{ADV}} \underbrace{-\left(\mathbf{u}_{x} \cdot \nabla\right) u-\left(\mathbf{u}_{y} \cdot \nabla\right) v}_{\mathrm{CT}} \underbrace{-\frac{1}{\rho_{0}} \nabla_{h}^{2} p^{\prime}}_{\mathrm{PRES}}+\underbrace{\nabla_{h} \cdot \mathbf{D}_{h}}_{\mathrm{TURB}}+\underbrace{f_{0} \zeta}_{\mathrm{COR}},
$$

where $\mathbf{u}=(u, v, w)$ is the fluid velocity, $\zeta$ is the relative vertical vorticity, $\rho_{0}=1 \mathrm{~kg} \mathrm{~m}^{-3}$ is a reference air density, $p^{\prime}$ is the perturbation pressure relative to the base state, $\mathbf{D}_{h}$ is the horizontal turbulent mixing vector [as described in Skamarock et al. (2008)], subscripts $x, y, z$, and $t$ denote derivatives, and the subscript $h$ indicates "horizontal." On the right-hand side is advection of $\delta$ (ADV), advection of $u$ and $v$ by the $x$ - and $y$-wind gradients [here denoted the "cross terms" (CT)], the negative horizontal Laplacian of the horizontal pressure perturbation (PRES), the divergence of turbulent momentum tendencies (TURB), and the Coriolis forcing (COR). Negative values of these terms act to strengthen convergence (or weaken divergence), and positive values of the terms act to strengthen divergence (or weaken convergence).

Although the physical interpretation of ADV is straightforward, some of the other terms of (4) merit additional explanation. CT modifies $\delta$ through selfadvection (of $u_{x}$ and $v_{y}$ ) and tilting of horizontal and vertical wind shears into horizontal confluence. PRES tends to force convergence into local pressure minima and divergence out of local pressure maxima, and TURB tends to force convergence upstream, and divergence downstream, of turbulent decelerations (and vice versa for turbulent accelerations). Finally, COR 

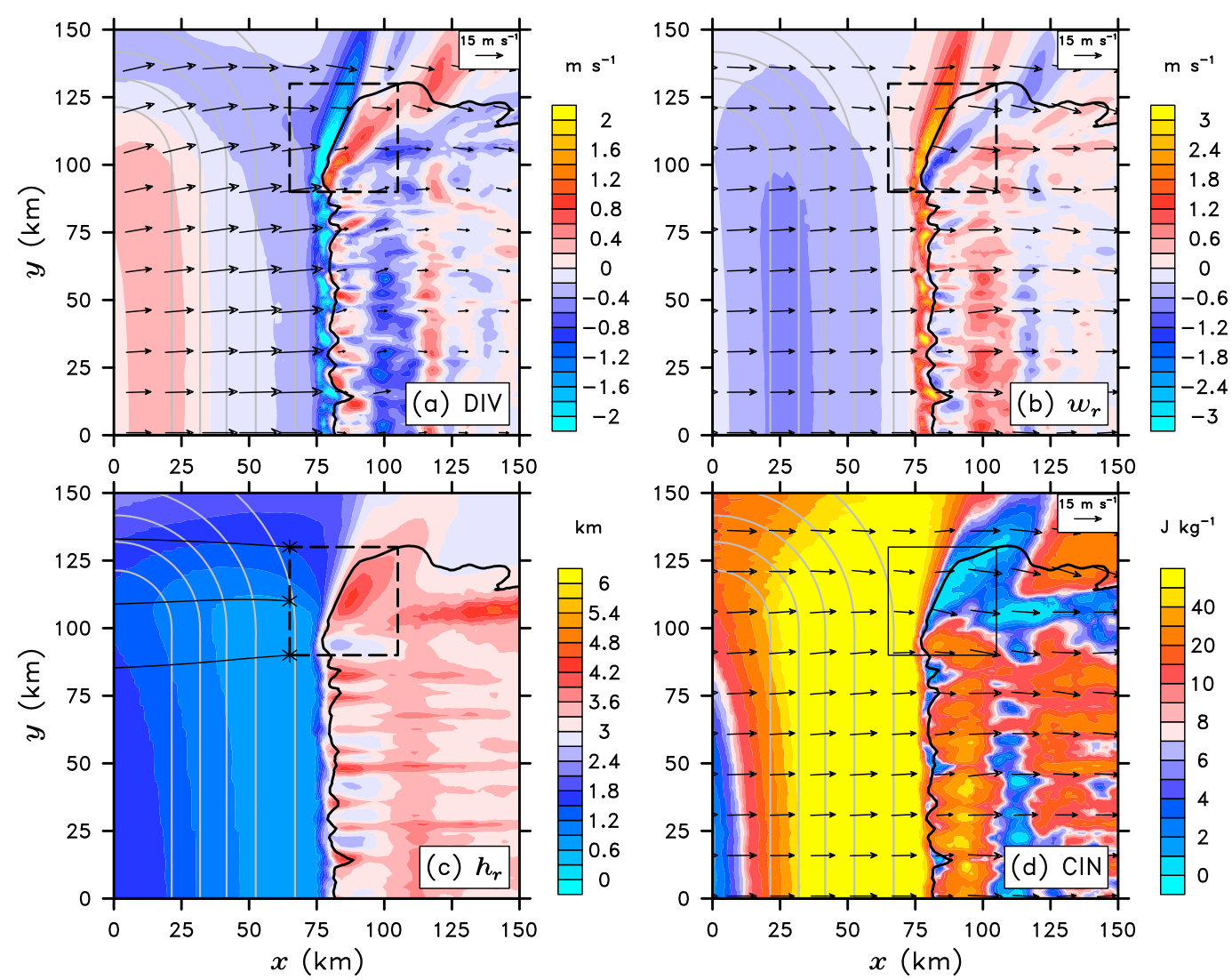

FIG. 11. Various quantities related to leeside vertical motion in the LAY36 simulation, including (a) vertically integrated horizontal divergence and mean horizontal wind vectors over the sub-band layer (DIV; filled), (b) vertical velocity $w_{r}$ (filled) and horizontal wind vectors at $h_{r}$, (c) $h_{r}$ and selected back streamlines of air parcels that arrive at the upstream edge of the boxed region, and (d) most unstable CIN and horizontal wind vectors at $h_{r}$. All panels are averaged over $6-9 \mathrm{~h}$, and the mountain wake (defined by the $u<8 \mathrm{~m} \mathrm{~s}^{-1}$ contour at $z=750 \mathrm{~m}$ ) is outlined by the thin black line. The boxed region in all panels denotes the area of convective band initiation, and terrain is shown in thin solid gray contours at 250-m intervals.

modulates $\delta$ by producing convergent (and unbalanced) inertial accelerations. In the Northern Hemisphere, for example, the Coriolis force points inward within anticyclones $(\zeta<0)$. In the absence of an opposing pressuregradient force, $\mathrm{COR}$ tends to generate convergence at the anticyclone center.

The right-hand-side terms of (4) are vertically integrated over the sub-band layer and averaged in time over 6-9h. Because the flow near the hydraulic jump is quasi-steady over this period, the sum of these terms is much less than the magnitude of the dominant terms (not shown). In the westerly prevailing flow of LAY36, ADV is generally positive on the western side, and negative on the eastern side, of the hydraulic jump (Figs. 12a,b). CT exhibits a similar pattern over the ridge midsection, largely due to the sum of zonal self-advection $\left(-u_{x}^{2}\right)$ and tilting $\left(-u_{z} w_{x}\right)$ terms. The former is always negative but the latter can be positive or negative. Zonal deceleration within the wake enhances the positive forward shear $u_{z}$, which is tilted into zonal diffluence $(\mathrm{CT}>0)$ and confluence $(\mathrm{CT}<0)$ along the western and eastern flanks of the hydraulic jump, respectively. Over the ridge ends, CT becomes more complex due to significant meridional winds and wind shear. PRES works in the opposite direction of $\mathrm{ADV}$ and $\mathrm{CT}$, favoring convergence on the western side of the jump where $p^{\prime}$ reaches a local minimum and divergence on the eastern side of the jump where the pressure gradient slackens.

Given that TURB is negligible (Fig. 12d) and COR = 0 in the nonrotating LAY36 case, PRES constitutes the key forcing for convergence within the hydraulic jump. As indicated by the mean sub-band pressure gradient vectors overlaid on Fig. 12c, the midsection of the leeside convergence line is forced primarily by converging zonal pressure gradients. By contrast, along the ridge ends, converging zonal and meridional pressure gradients combine to enhance the forcing for ascent. The meridional component stems from the mountain-wave 

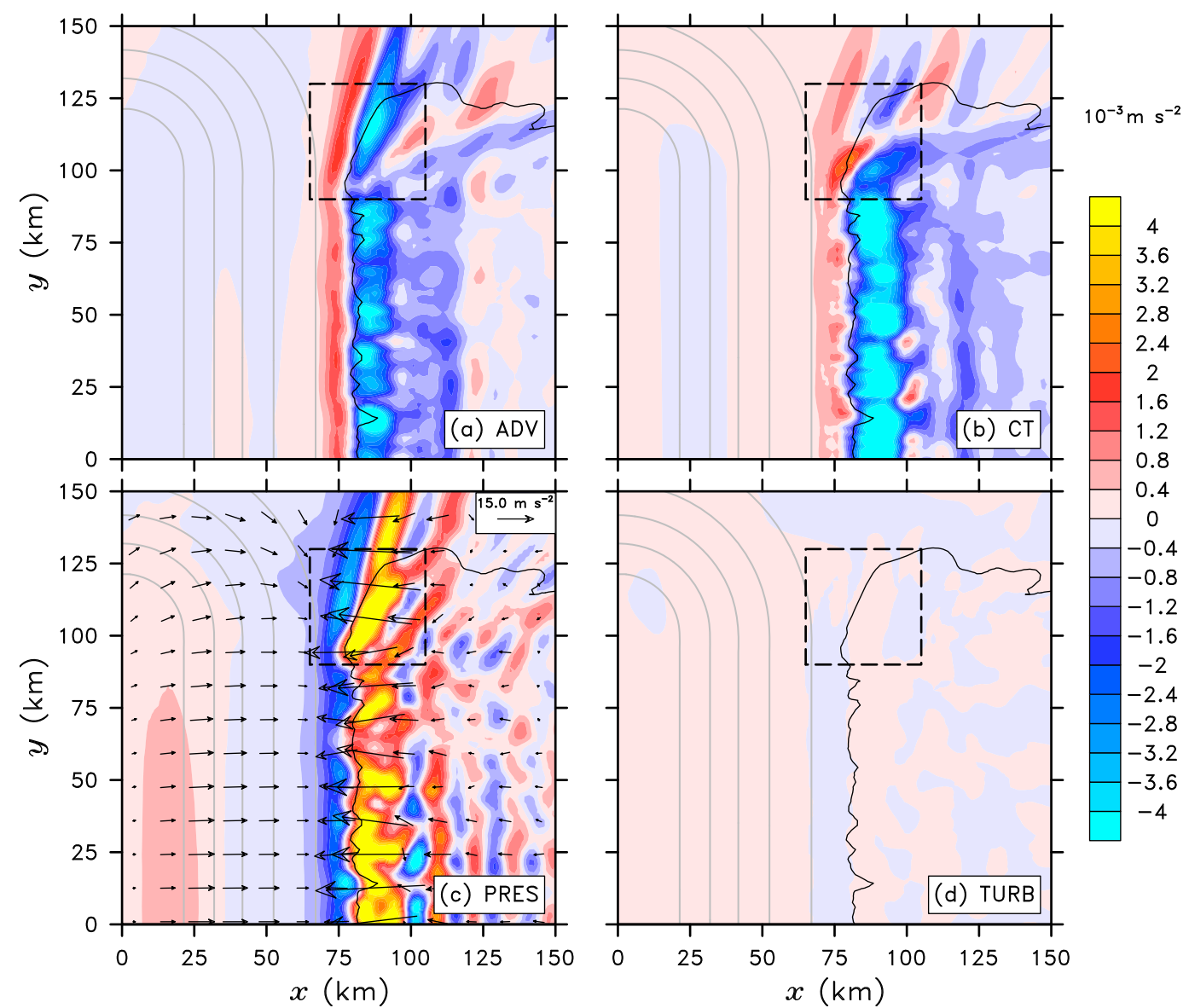

FIG. 12. Terms of the horizontal divergence budget [(4)] of the LAY36 simulation, integrated from $h_{s}$ to $h_{r}$ and time-averaged over 6-9 h: (a) advection (ADV), (b) cross terms (CT), (c) pressure forcing (PRES), with layer-mean pressure gradients shown by vectors, and (d) turbulence (TURB). As in Fig. 11, the mountain wake is outlined by the thin black contour, the dashed boxed region denotes the area of convective band initiation, and terrain is shown in thin gray contours at 250-m intervals.

pressure minimum over the lee slope, which is strongest past the ridge midsection and gradually weakens to the north and south. The resulting meridional pressure gradients are generally directed toward the ridge midsection and reach their maximum intensity within the boxed region. This forcing maintains the aforementioned strip of sub-band convergence over the ridge ends (Fig. 11a) and enhances the convergence within the boxed region.

Unlike the symmetric edge bands in LAY36, the northern edge band is favored (and the southern band suppressed) in LAY36-COR (cf. Figs. 7a-c and 7d-f). This asymmetry is reflected in the horizontal divergence, $w_{r}$, and $h_{r}$ fields in Fig. 13. Although the layer-integrated convergence and $w_{r}$ are larger near the southern end of the hydraulic jump (Figs. 13a,b), the flow traversing the northern end exhibits more trajectory-integrated convergence on its journey from the mountain crest to the hydraulic jump. As a result, it undergoes more upward displacement, and more readily eliminates the convective inhibition, within the hydraulic jump (Figs. 13c,d).

Among all of the terms in (4), the one that differs the most between the northern and southern ridge ends is COR (Fig. 14) (TURB is again negligible and not shown). COR is negative past the northern ridge end and positive past the southern ridge end, due to opposing signs of orographically generated relative vorticity (e.g., Smolarkiewicz et al. 1988). Although this forcing over the ridge ends is small in magnitude, its contributions are important. For example, if air traveling at $15 \mathrm{~m} \mathrm{~s}^{-1}$ traversed the $\sim 60$-km-wide region of negative COR over the northern ridge end (with a magnitude of $\sim 6 \times 10^{-5} \mathrm{~m} \mathrm{~s}^{-2}$ ), its depth-integrated convergence would increase by $0.3 \mathrm{~m} \mathrm{~s}^{-1}$, which is comparable in magnitude to the depth-integrated convergence itself (Fig. 13a). COR also exhibits a pronounced minimum within the boxed region on the northern end and maximum within the boxed region on the southern end, 

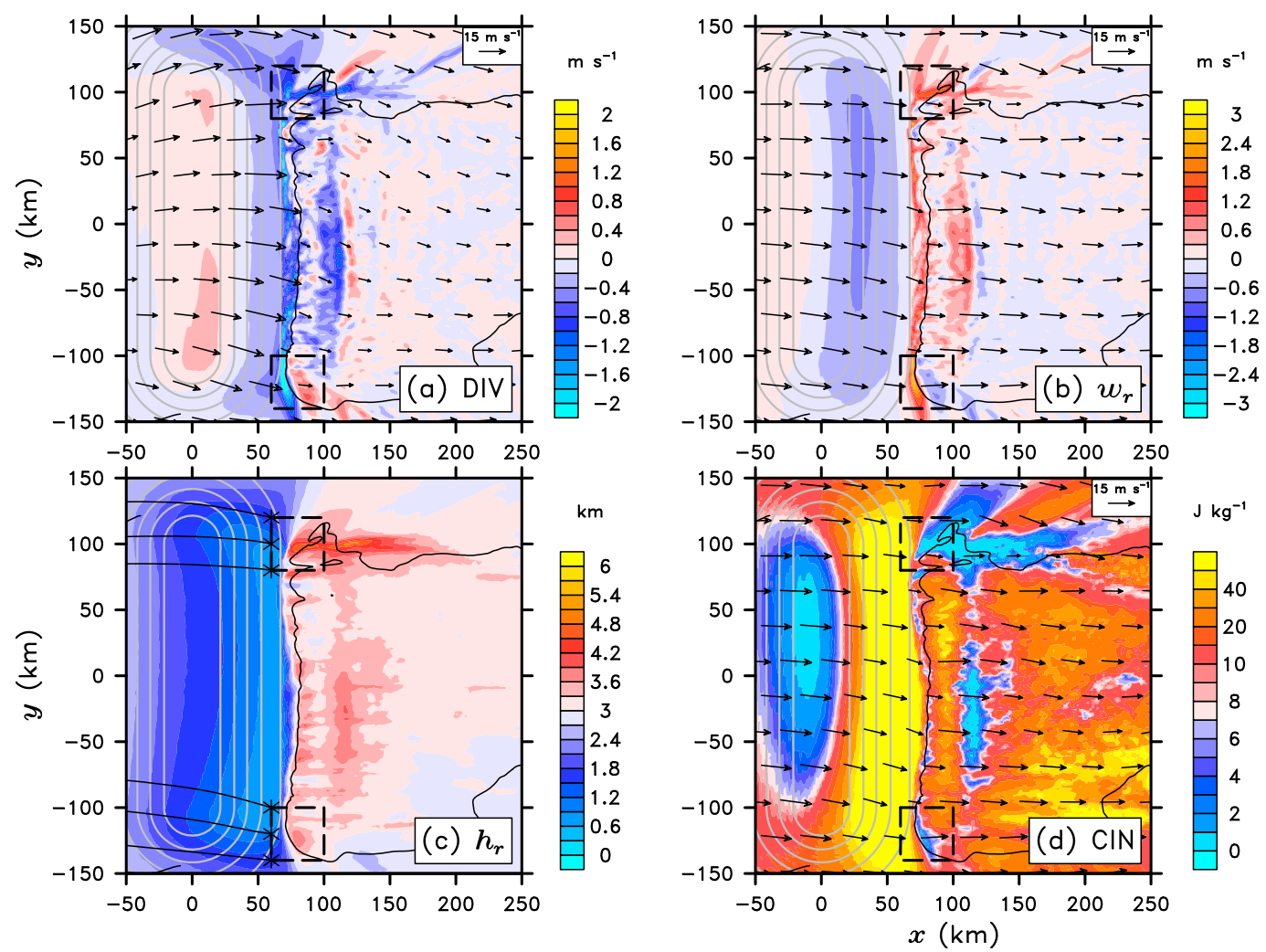

FIG. 13. As in Fig. 11, but for the LAY36-COR simulation.

adjacent to a similarly located minimum of PRES (cf. Figs. 12c and 12d). Thus, past the northern ridge end, COR acts to strengthen the forcing for ascent and favor band initiation, and vice versa past the southern end.

Similar analyses of the divergence budget have also been applied to the uniformly stratified N012, N012-T303, and N011-T303 simulations, where the edge bands were either absent or short lived (not shown). The leeside pressure forcing is greatly reduced in these cases, which limits the corresponding horizontal convergence and vertical motion. Compared to LAY36 and LAY36-COR, the leeside low-level updrafts in N011-T303 are weakened by $\sim 50 \%$, as are the corresponding displacements in $h_{r}$. Moreover, in N011-T303, the location of the maximum leeside displacement shifts from past the ridge edges $(6-9 \mathrm{~h})$ to past the ridge midsection (9-12 h). This time variation may stem from a gradual invigoration of windward-side convection (Figs. 6d-f) progressively damping the mountain-wave-induced pressure gradients in the lee.

\section{Other sensitivities}

The environmental conditions and terrain forcing considered thus far represent a very small sampling of midlatitude parameter space. Moreover, only a single grid resolution has been considered, which may be too coarse to accurately resolve individual convective cells (e.g., Bryan et al. 2003). In this section, we vary the terrain configuration and horizontal grid resolution to evaluate the robustness of the simulated bands. For simplicity, all of these simulations omit the Coriolis force.

\section{a. Ridge height}

The ridge height considered thus far $\left(h_{m}=1.5 \mathrm{~km}\right)$ is representative of many midlatitude mountain ranges, but not all of them. Notably, the two downwind-band cases that inspired this study formed past the Alps, with a characteristic $h_{m} \approx 2.5 \mathrm{~km}$. To evaluate the sensitivity of the downwind bands to $h_{m}$, we conduct two simulations identical to LAY36 except that $h_{m}$ is decreased by $50 \%$ to $0.75 \mathrm{~km}$ in HM750 and increased by $50 \%$ to $2.25 \mathrm{~km}$ in HM2250. As reflected by their effects on $M$ (Table 1 ), these variations in $h_{m}$ promote decreased upstream blocking in HM750 and increased upstream blocking in HM2250. At $9 \mathrm{~h}$ of model integration, reduced forcing for leeside ascent in HM750 is incapable of generating downwind bands (Fig. 15a). By contrast, two bands similar to those in LAY36 develop in HM2250 (Fig. 15b). Although the latter suggests that 

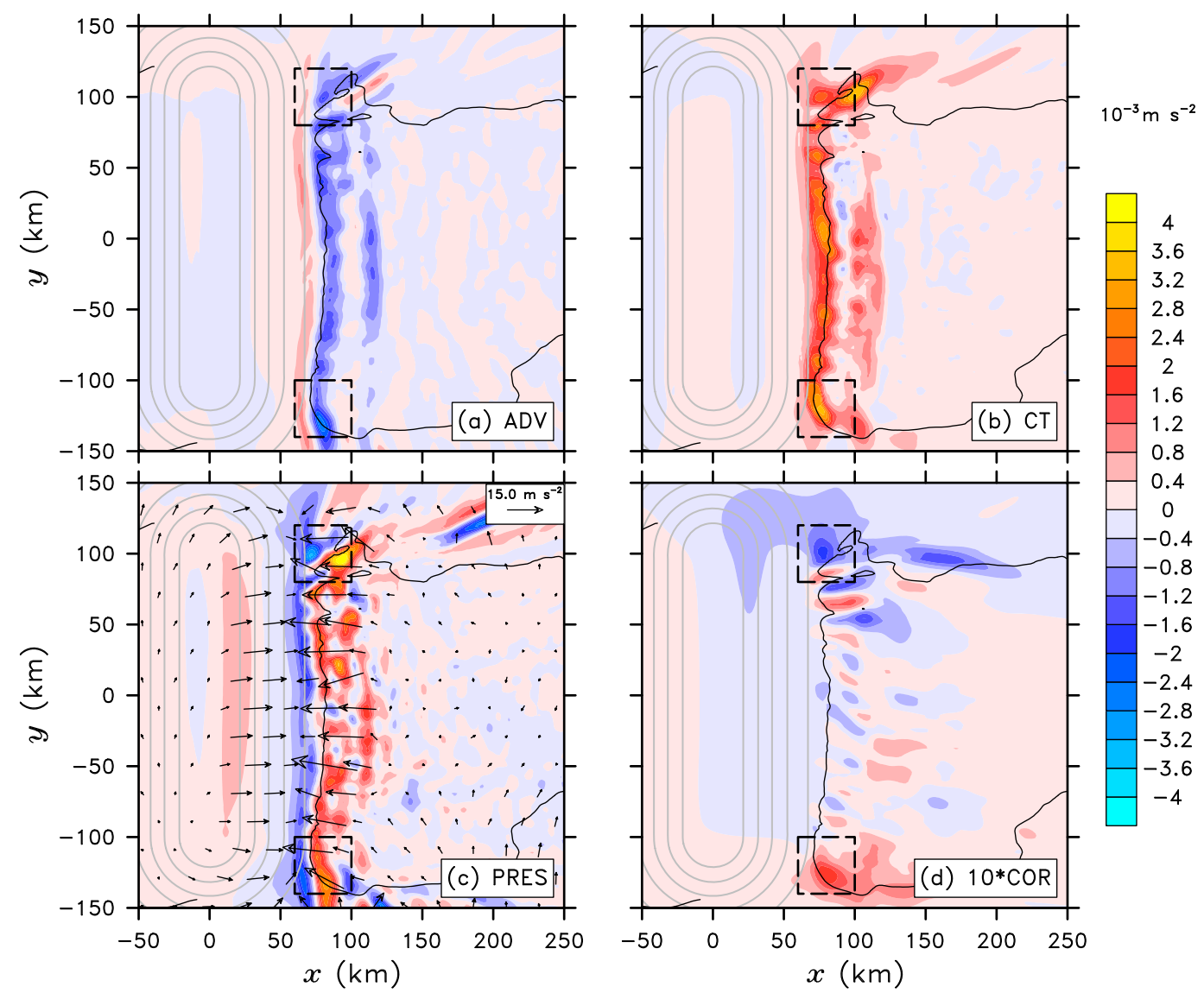

FIG. 14. As in Fig. 12, but for the LAY36-COR simulation. Instead of showing the TURB term in (4), (d) shows the COR term multiplied by a factor of 10 .

band formation is robust over taller ridges, the two edge bands in HM2250 vanish by $12 \mathrm{~h}$ (not shown). Preliminary analysis suggests that a gradual increase in upstream blocking acts to reduce the depth of the sub-band layer crossing the ridge, which shifts the hydraulic jump upstream and weakens it. Thus, at least for the environmental conditions in LAY36, medium-height ridges are more favorable for long-lived bands than taller ridges.

\section{b. Small-scale terrain}

As opposed to the smooth ridge used thus far, real mountain ranges are rugged, with large-amplitude terrain variations that may influence band formation. To examine such complex terrain, we perform two simulations where different fields of small-scale terrain $h_{\mathrm{ss}}$ are added to the smooth profile in (1). The first (GAP) cuts a simple Gaussian gap of depth $h_{m} / 2$ though the ridge center at $y=0$ :

$$
h_{\mathrm{ss}}=-\frac{h_{m}}{2} \exp \left[-\left(\frac{x}{a}\right)^{2}-\left(\frac{y}{a / 2}\right)^{2}\right],
$$

where $h_{m}$ and $a$ are the same as in (1). The second (K53) uses a random noise field, constructed by defining a zero-mean perturbation field with a $\kappa^{-5 / 3}$ power spectrum, where $\kappa=\sqrt{k^{2}+l^{2}}$ and $k$ and $l$ are the $x$ and $y$ wavenumbers. Such a spectrum is characteristic not only of 3D mesoscale turbulence (e.g., Nastrom and Gage 1985), but also of 2D mesoscale terrain variations (e.g., Kirshbaum et al. 2007b). The amplitude spectrum is defined in wavenumber space, with a shortwave cutoff at $\kappa=6 \Delta x$ to eliminate forcing at poorly resolved scales, and inverted into physical space with a random phase spectrum. The field is then scaled to have a maximum amplitude of $h_{m} / 2$ and multiplied by $h / h_{m}$ in (1) so that it decays away from the ridge.

Along with two edge bands, a third "gap" band past the central gap develops in GAP (Fig. 15c). A similar analysis as that performed in section 4 reveals that the sub-band convergence and $w_{r}$ are both maximized past the gap (Figs. 16a,b). These maxima form about $25 \mathrm{~km}$ farther east than corresponding maxima to the north and south, suggesting that hydraulic-jump formation is delayed past the gap. This delay may stem from an 

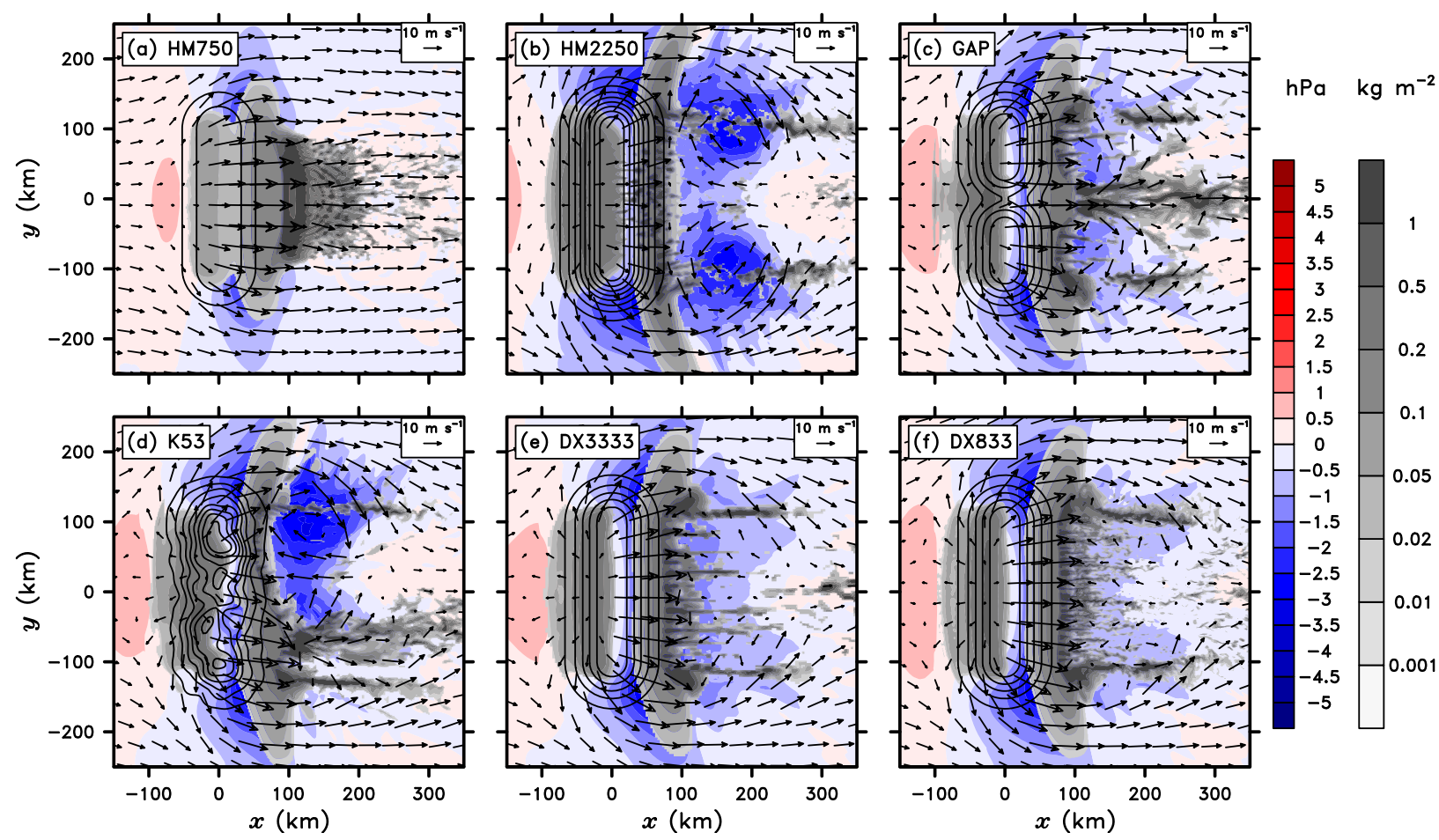

FIG. 15. Lowest-model-level pressure perturbation (color fill), wind vectors, and CWP (gray fill) at $9 \mathrm{~h}$ for various sensitivity tests based on the LAY36 case: (a) HM750, (b) HM2250, (c) GAP, (d) K53, (e) DX3333, and (f) DX833. Terrain is shown in solid black contours every $250 \mathrm{~m}$.

increased depth of the sub-band layer channeled through the gap, followed by a less rapid thinning of this layer over the relatively shallow lee slope (Fig. 16c). Because perturbation pressure increases rapidly across the hydraulic jump, meridional variations in the $x$ location of the jump give rise to meridional pressure gradients (Fig. 16d). These gradients converge with zonal pressure gradients within the boxed region to locally enhance the forcing for ascent. Given that this forcing acts on an already deeper sub-band layer than that to the north and south, very large upward displacements are found within the boxed region.

A third band also develops in K53 (Fig. 15d), just to the north of the southern ridge end (at $y \approx-70 \mathrm{~km}$ ). Similar to the gap band in GAP, this band forms past a deep gap in the rugged terrain (centered at $y \approx-20 \mathrm{~km}$ ). The meridional displacement of around $50 \mathrm{~km}$ between the gap location and the band initiation point stems from the gap alignment: rather than aligning zonally with the flow, it aligns from northwest to southeast. The flow channeled through this gap adopts this same alignment, and thus travels some distance to the south before developing a leeside hydraulic jump.

\section{c. Horizontal grid spacing}

At a horizontal grid spacing of $\Delta_{h}=5$ and $1.67 \mathrm{~km}$ on the outer and inner nests, respectively, downwind bands with horizontal widths of $\sim 10 \mathrm{~km}$ are only marginally resolved. To evaluate the sensitivity of these bands to $\Delta_{h}$, we perform two additional simulations where $\Delta_{h}$ is doubled and halved (on both model grids) to respective values of $\Delta_{h}=10$ and $3.33 \mathrm{~km}(\mathrm{DX} 3333)$ and $\Delta_{h}=2.5$ and $0.83 \mathrm{~km}$ (DX833). Despite these major changes in grid resolution, the basic characteristics of the original LAY36 simulation are retained: two longitudinal edge bands form past the ridge with zonal lengths of around $200 \mathrm{~km}$. Thus, the basic findings of the above simulations appear robust to changes in $\Delta_{h}$, provided it is sufficiently small to explicitly represent the bands.

\section{Comparison to observed events}

Are the downwind bands simulated herein comparable to those observed in nature? In the Alpine event shown in Fig. 1, two bands (B1 and B2) formed past deep valleys in the lee of the Alps, with B1 lying close to the western (left, relative to the incoming flow) edge of the Alps. Given these locations, and the similarity of the upstream flow in this event to that in LAY36, these bands may indeed owe their existence to a similar mechanism as the edge bands in LAY36 and LAY36COR (band B1) and/or the gap bands in GAP and K53.

The absence of upstream moist instability in the Siedersleben and Gohm (2016) case study suggests a 

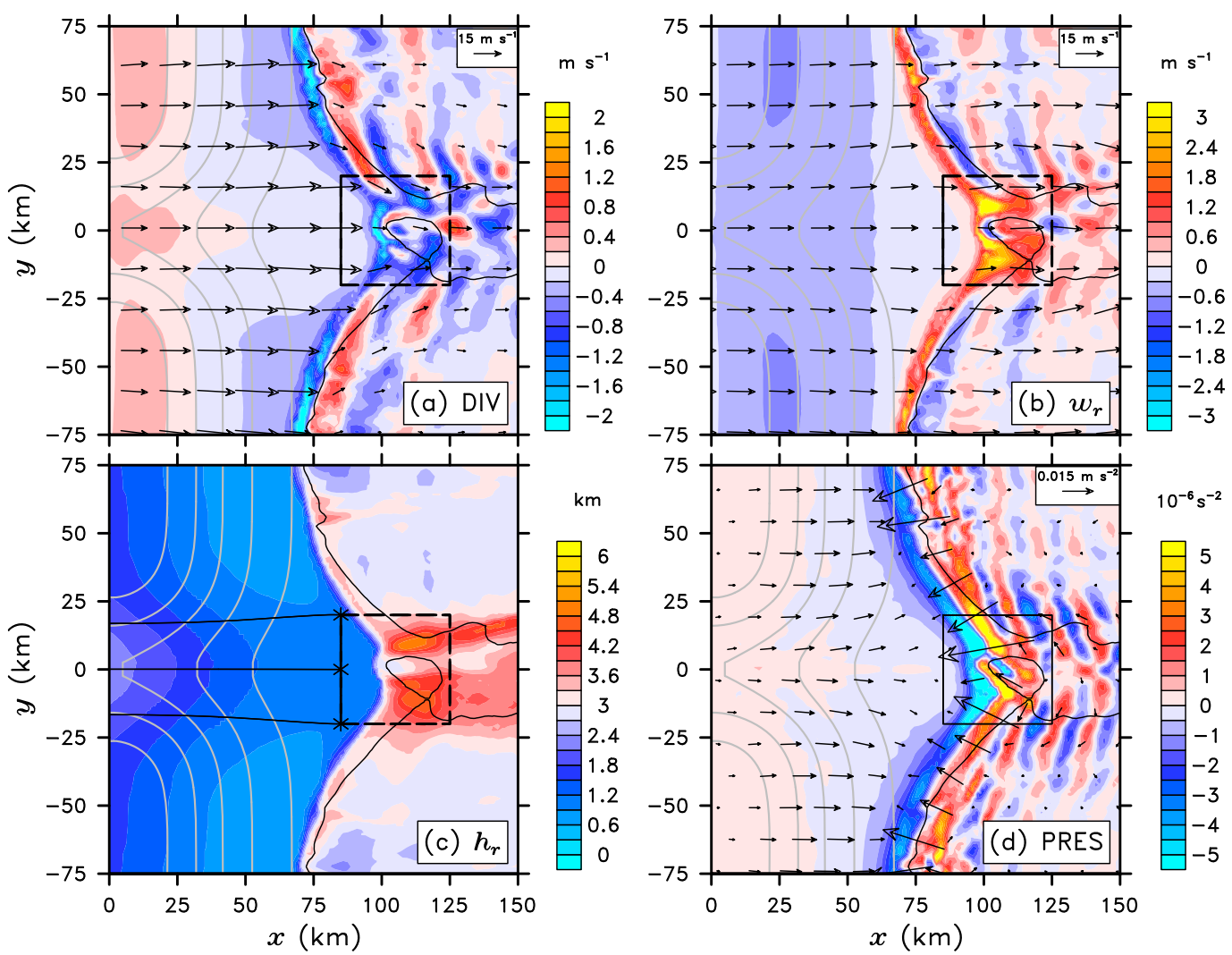

FIG. 16. Various quantities related to leeside vertical motion in the GAP simulation, including (a) vertically integrated horizontal divergence and mean horizontal wind vectors over the sub-band layer (DIV; filled), (b) vertical velocity $w_{r}$ (filled) and horizontal wind vectors at $h_{r}$, (c) $h_{r}$ and selected back streamlines of air parcels that arrive at the upstream edge of the boxed region, and (d) pressure forcing (PRES), with layer-mean pressure gradients shown by vectors. As in Fig. 11, the mountain wake is outlined by the thin black contour, the boxed region denotes the area of convective-band initiation, and terrain is shown in thin gray contours at $250-\mathrm{m}$ intervals.

different mechanism for band formation than the convective one proposed here, and they attributed their bands to the release of inertial instability within negative orographic PV banners. However, it is conceivable that moist convection played a more important role than that hypothesized by Siedersleben and Gohm (2016). As previously mentioned, their upstream sounding (their Fig. 6a) possessed a subfreezing midlevel layer that was nearly moist neutral with respect to liquid water, and thus was likely marginally unstable with respect to ice. Moreover, their sounding was taken $3 \mathrm{~h}$ before the bands developed, and the atmosphere may have destabilized during the intervening period as a frontal system approached. Because their study focused on the eastern Alps, Siedersleben and Gohm (2016) did not evaluate the existence of a left edge band, and the relationship between the downwind bands and gaps in the Alpine terrain in their Fig. 1 is not obvious. Thus, the relevance of the physical mechanism(s) proposed herein to the Siedersleben and Gohm (2016) case is unclear.
In addition, the underlying mechanisms of the bands simulated herein probably differ from those of the "minor" downwind bands observed by Schumacher et al. (2010). As mentioned in section 1, the upstream flow in Schumacher et al. (2010) consisted of a deep layer of nearly dry-neutral flow with $M \ll 1$ and no elevated inversion layer, suggesting minimal mountainwave activity at lower levels. Thus, hydraulic-jump formation was unlikely, and the leeside mountain-wave forcing for ascent may have been insufficient to initiate moist convection. A more plausible, but still speculative, explanation for those bands is an interaction of instabilities-inertial instabilities within leeside PV banners may have provided the necessary lifting to initiate shallow moist convection.

In our rotating simulations, inertial instability was generated over and to the lee of the ridge by mesoscale mountain-wave forcing (e.g., Figs. 5d,e,f and 7d,e,f). Similarly, inertial instability can arise from negative PV banners downwind of small-scale topographic features 

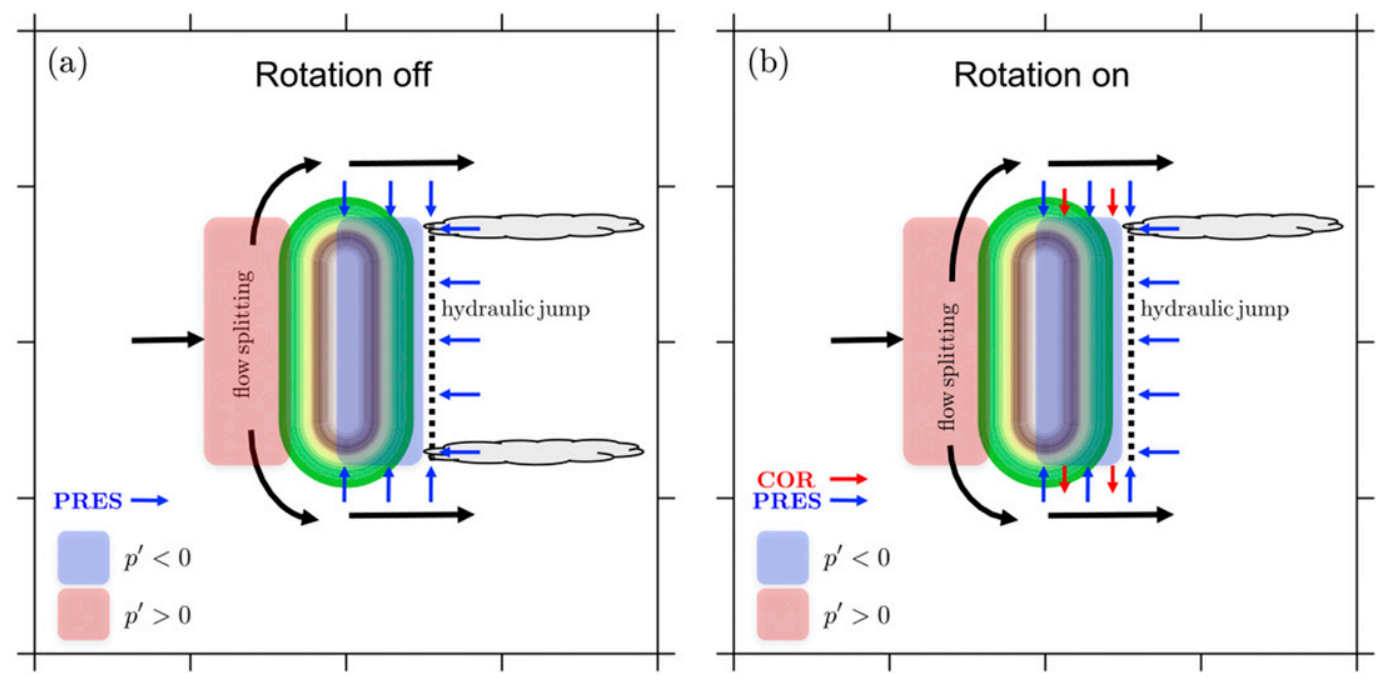

FIG. 17. Schematic of the processes giving rise to edge bands for (a) nonrotating flow (symmetric edge bands) and (b) rotating flow (preferred left edge band). The arrows represent the directions of relevant leeside horizontal accelerations, with the convergence (divergence) of these vectors representing forcing for ascent (descent).

(e.g., Siedersleben and Gohm 2016) or from moist convection itself (e.g., Holt and Thorpe 1991; Schultz and Knox 2007). Such cases with orographically generated inertial instability should be distinguished from those where the upstream flow already contains inertial instability (e.g., Schumacher et al. 2010). In the latter, the mountain ridge may act more as a mechanism for initiating unstable motions than a mechanism for generating the instability.

\section{Conclusions}

Recent observations suggest that quasi-stationary convective bands, some capable of producing heavy precipitation, may form past midlatitude mountain ridges. To gain insight into the environmental conditions and physical mechanisms behind such "downwind" bands, convection-permitting idealized numerical simulations with WRF were conducted. A sampling of environmental parameter space suggests that upstream flows with a multilayer stability structure, including a strongly stable surface-based layer topped by a weakly stable midlevel layer, are favorable for band development. In such environments, the flow responds quasi-hydraulically to significant terrain obstacles, with a leeside hydraulic jump featuring strong, upright ascent. When the midlevel layer is moist and conditionally and/or potentially unstable, this ascent may initiate elevated cumulus convection.

The processes responsible for initiating the downwind bands are illustrated in the schematic of Fig. 17. In nonrotating flow over smooth ridges, two downwind bands form in the lee, one past each ridge end. These locations are favorable for band initiation because the air parcels traversing them undergo more trajectoryintegrated uplift than those traversing the central section of the ridge. This enhanced ascent stems primarily from meridional pressure gradients in the sub-band layer associated with gradients in terrain height over the ridge ends (Fig. 17a). This meridional forcing limits parcel descent over the lee slope and enhances parcel ascent within the hydraulic jump. In rotating flow, the Coriolis force tends to enhance the left band and suppress the right band (from the perspective of the incoming flow), suggesting that the former are favored in the midlatitude Northern Hemisphere. This asymmetry stems from the opposite-signed relative-vorticity perturbations past the northern and southern ridge ends, which give rise to locally enhanced convergence and divergence, respectively (Fig. 17b).

Some other notable sensitivities of the simulated bands include the following:

- Moist instability was a necessary condition for band formation. Orographically generated inertial instability, which developed within the mountain lee of the rotating simulations, played at most a secondary role.

- Deeper and/or stronger midlevel moist instability gave rise to stronger downwind bands and heavier precipitation.

- As with the "upwind" bands studied by Kirshbaum et al. (2007a), vertical shear of the basic-state horizontal wind was not required to organize the bands.

- The simulated bands were the most persistent over medium-height (1.5-km high) ridges, which produced 
stronger leeside uplift than that over taller or shorter ridges.

- Over ridges with sub-ridge-scale terrain variations, additional bands developed downstream of deep valleys or gaps in the terrain, again due to a local superposition of zonal and meridional pressure gradients.

The downwind bands simulated herein, and the physical mechanism behind them, represent just one of several types of cloud bands past mesoscale terrain. Past studies have investigated convective bands that form downstream of convergent mountains wakes (e.g., Mass 1981; Barrett et al. 2015), within broad regions of inertial or symmetric instability (e.g., Schultz and Knox 2007; Schumacher et al. 2010), and within orographically generated and inertially unstable PV banners (Siedersleben and Gohm 2016). Therefore, caution is advised when attributing downwind bands to specific physical mechanisms. Further investigation is required to precisely distinguish the environmental and terrain-related parameters favoring each band type. Moreover, the numerical setup in this study was highly idealized, which constitutes a reasonable first step but should be improved upon in future work.

Acknowledgments. We are grateful to Jonathan Fairman Jr. and Alexander Gohm for their physical insights and contributions to the analysis. We also thank European Meteorological Services Network (EUMETNET) for providing the OPERA pan-European radar composites used in Fig. 1. The contributions of DJK were funded by the Natural Science and Engineering Research Council (NSERC) Grant NSERC/RGPIN 418372-17 and by the Canada Foundation for Innovation through the Leaders Opportunity Fund Grant 30674. Numerical simulations were performed on the Guillimin supercomputer at McGill University, under the auspices of Calcul Québec and Compute Canada. Partial funding for DMS was provided by the Natural Environment Research Council Grant NE/1024984/1 to the University of Manchester through the Precipitation Structures over Orography (PRESTO) project.

\section{REFERENCES}

Andretta, T. A., and D. S. Hazen, 1998: Doppler radar analysis of a Snake River Plain convergence event. Wea. Forecasting, 13, 482-491, https://doi.org/10.1175/1520-0434(1998)013<0482: DRAOAS $>2.0 . \mathrm{CO} ; 2$.

Asai, T., 1970: Three-dimensional features of thermal convection in a plane Couette flow. J. Meteor. Soc. Japan, 48, 18-29, https://doi.org/10.2151/jmsj1965.48.1_18.

Barrett, A. I., S. L. Gray, D. J. Kirshbaum, N. M. Roberts, D. M. Schultz, and J. G. Fairman, 2015: Synoptic versus orographic control on stationary convective banding. Quart. J. Roy. Meteor. Soc., 141, 1101-1113, https://doi.org/10.1002/qj.2409.
Bryan, G. H., J. C. Wyngaard, and J. M. Fritsch, 2003: Resolution requirements for the simulation of deep moist convection. Mon. Wea. Rev., 131, 2394-2416, https://doi.org/ 10.1175/1520-0493(2003)131<2394:RRFTSO > 2.0.CO;2.

Cosma, S., E. Richard, and F. Miniscloux, 2002: The role of small-scale orographic features in the spatial distribution of precipitation. Quart. J. Roy. Meteor. Soc., 128, 75-92, https://doi.org/10.1256/ 00359000260498798

Durran, D. R., 1986: Another look at downslope windstorms. Part I: The development of analogs to supercritical flow in an infinitely deep, continuously stratified fluid. J. Atmos. Sci., 43, 2527-2543, https://doi.org/10.1175/1520-0469(1986)043<2527: ALADWP $>2.0 . \mathrm{CO} ; 2$.

— Part II: Nonlinear amplification beneath wave-overturning layers. J. Atmos. Sci., 44, 3402-3412, https://doi.org/10.1175/ 1520-0469(1987)044<3402:ALADWP>2.0.CO;2.

Galewsky, J., 2008: Orographic clouds in terrain-blocked flows: An idealized modeling study. J. Atmos. Sci., 65, 3460-3478, https:// doi.org/10.1175/2008JAS2435.1.

Holt, M. W., and A. J. Thorpe, 1991: Localized forcing of slantwise motion at fronts. Quart. J. Roy. Meteor. Soc., 117, 943-963, https://doi.org/10.1002/qj.49711750104.

Holton, J. R., 1972: An Introduction to Dynamic Meteorology. Academic Press, 319 pp.

Hong, S.-Y., J. Dudhia, and J.-O. J. Lim, 2006: The WRF singlemoment 6-class microphysics scheme (WSM6).J. Kor. Meteor. Soc., 42, 129-151.

Huuskonen, A., E. Saltikoff, and I. Holleman, 2014: The operational weather radar network in Europe. Bull. Amer. Meteor. Soc., 95, 897-907, https://doi.org/10.1175/BAMS-D-12-00216.1.

Janjić, Z. I., 1994: The step-mountain eta coordinate model: Further developments of the convection, viscous sublayer, and turbulence closure schemes. Mon. Wea. Rev., 122, 927-945, https://doi.org/ 10.1175/1520-0493(1994)122<0927:TSMECM > 2.0.CO;2.

Kirshbaum, D. J., and D. R. Durran, 2005a: Atmospheric factors governing banded orographic convection. J. Atmos. Sci., 62, 3758-3774, https://doi.org/10.1175/JAS3568.1.

$\longrightarrow$, and — 2005b: Observations and modeling of banded orographic convection. J. Atmos. Sci., 62, 1463-1479, https:// doi.org/10.1175/JAS3417.1.

— G. H. Bryan, R. Rotunno, and D. R. Durran, 2007a: The triggering of orographic rainbands by small-scale topography. J. Atmos. Sci., 64, 1530-1549, https://doi.org/10.1175/JAS3924.1.

, R. Rotunno, and G. H. Bryan, 2007b: The spacing of orographic rainbands triggered by small-scale topography. J. Atmos. Sci., 64, 4222-4245, https://doi.org/10.1175/2007JAS2335.1.

- T. M. Merlis, J. R. Gyakum, and R. McTaggart-Cowan, 2018: Sensitivity of idealized moist baroclinic waves to environmental temperature and moisture content. J. Atmos. Sci., $\mathbf{7 5}$, 337-360, https://doi.org/10.1175/JAS-D-17-0188.1.

Mass, C., 1981: Topographically forced convergence in western Washington state. Mon. Wea. Rev., 109, 1335-1347, https://doi. org/10.1175/1520-0493(1981)109<1335:TFCIWW>2.0.CO;2.

Miniscloux, F., J. D. Creutin, and S. Anquetin, 2001: Geostatistical analysis of orographic rainbands. J. Appl. Meteor., 40, 18351854, https://doi.org/10.1175/1520-0450(2001)040<1835: GAOOR $>2.0 . \mathrm{CO} ; 2$.

Nastrom, G. D., and K. S. Gage, 1985: A climatology of atmospheric wavenumber spectra of wind and temperature observed by commercial aircraft. J. Atmos. Sci., 42, 950960, https://oi.org/10.1175/1520-0469(1985)042<0950:ACOAWS >2.0. $\mathrm{CO} ; 2$. 
Pierrehumbert, R. T., and B. Wyman, 1985: Upstream effects of mesoscale mountains. J. Atmos. Sci., 42, 977-1003, https://doi.org/ 10.1175/1520-0469(1985)042<0977:UEOMM>2.0.CO;2.

Scheffknecht, P., E. Richard, and D. Lambert, 2016: A highly localized high-precipitation event over Corsica. Quart. J. Roy. Meteor. Soc., 142 (S1), 206-221, https://doi.org/ 10.1002/qj.2795.

Schultz, D. M., and P. N. Schumacher, 1999: The use and misuse of conditional symmetric instability. Mon. Wea. Rev., 127, 27092732, https://doi.org/10.1175/1520-0493(1999)127<2709: TUAMOC $>2.0 . C O ; 2$; Corrigendum, 128, 1573.

— , and J. A. Knox, 2007: Banded convection caused by frontogenesis in a conditionally, symmetrically, and inertially unstable environment. Mon. Wea. Rev., 135, 2095-2110, https:// doi.org/10.1175/MWR3400.1.

Schumacher, R. S., D. M. Schultz, and J. A. Knox, 2010: Convective snowbands downstream of the Rocky Mountains in an environment with conditional, dry symmetric, and inertial instabilities. Mon. Wea. Rev., 138, 4416-4438, https://doi.org/ 10.1175/2010MWR3334.1.

- ——, and — 2015: Influence of terrain resolution on banded convection in the lee of the Rocky Mountains.
Mon. Wea. Rev., 143, 1399-1416, https://doi.org/10.1175/ MWR-D-14-00255.1.

Siedersleben, S. K., and A. Gohm, 2016: The missing link between terrain-induced potential vorticity banners and banded convection. Mon. Wea. Rev., 144, 4063-4080, https://doi.org/ 10.1175/MWR-D-16-0042.1.

Skamarock, W. C., and Coauthors, 2008: A description of the Advanced Research WRF version 3. NCAR Tech. Note NCAR/TN-475+STR, 125 pp.

Smith, R. B., 1989: Hydrostatic flow over mountains. Advances in Geophysics, Vol. 31, Academic Press, 1-41, https://doi.org/ 10.1016/S0065-2687(08)60052-7.

—, A. C. Gleason, P. A. Gluhosky, and V. Grubisisic, 1997: The wake of St. Vincent. J. Atmos. Sci., 54, 606-623, https://doi.org/ 10.1175/1520-0469(1997)054<0606:TWOSV>2.0.CO;2.

Smolarkiewicz, P. K., R. M. Rasmussen, and T. L. Clark, 1988: On the dynamics of Hawaiian cloud bands: Island forcing. J. Atmos. Sci., 45, 1872-1905, https://doi.org/10.1175/1520-0469(1988) 045<1872:OTDOHC $>2.0 . \mathrm{CO} ; 2$.

Thompson, C. F., D. M. Schultz, and G. Vaughan, 2018: A global climatology of tropospheric inertial instability. J. Atmos. Sci., 75, 805-825, https://doi.org/10.1175/JAS-D-17-0062.1. 